\title{
Leishmania donovani tyrosyl-tRNA synthetase structure in complex with a tyrosyl adenylate analog and comparisons with human and protozoan counterparts
}

Ximena Barros-Álvarez ${ }^{1,2}$, Keshia M. Kerchner ${ }^{1}$, Cho Yeow Koh ${ }^{1, *}$, Stewart Turley ${ }^{1}$, Els Pardon $^{3,4}$, Jan Steyaert ${ }^{3,4}$, Ranae M. Ranade ${ }^{5}$, J. Robert Gillespie ${ }^{5}$, Zhongsheng Zhang ${ }^{1}$, Christophe L. M. J. Verlinde ${ }^{1}$, Erkang Fan ${ }^{1}$, Frederick S. Buckner ${ }^{5}$ and Wim G. J. Hol ${ }^{1 \#}$

${ }^{1}$ Department of Biochemistry, University of Washington, Seattle WA, USA; ${ }^{2}$ Laboratorio de Enzimología de Parásitos, Facultad de Ciencias, Universidad de los Andes, Mérida, Venezuela;

${ }^{3}$ Structural Biology Brussels, Vrije Universiteit Brussel, Brussel, Belgium; ${ }^{4}$ VIB-VUB Center for Structural Biology, VIB, Brussels, Belgium; ${ }^{5}$ Division of Allergy and Infectious Diseases, School of Medicine, University of Washington, Seattle, WA, USA. * Current address: Department of Biological Sciences, National University of Singapore, Singapore 117543.

\# Corresponding author: wghol@u.washington.edu. 


\section{ABSTRACT}

The crystal structure of Leishmania donovani tyrosyl-tRNA synthetase (LdTyrRS) in complex with a nanobody and the tyrosyl adenylate analog TyrSA was determined at $2.75 \AA$ resolution. Nanobodies are the variable domains of camelid heavy chain-only antibodies. The nanobody makes numerous crystal contacts and in addition reduces the flexibility of a loop of LdTyrRS. TyrSA is engaged in many interactions with active site residues occupying the tyrosine and adenine binding pockets. The LdTyrRS polypeptide chain consists of two pseudomonomers, each consisting of two domains. Comparing the two independent chains in the asymmetric unit reveals that the two pseudo-monomers of $L d \mathrm{TyrRS}$ can bend with respect to each other essentially as rigid bodies. This flexibility might be useful in the positioning of tRNA for catalysis since both pseudo-monomers in the $L d$ TyrRS chain are needed for charging tRNA $^{\text {Tyr }}$.

An "extra pocket" (EP) appears to be present near the adenine binding region of LdTyrRS. Since this pocket is absent in the two human homologous enzymes, the EP provides interesting opportunities for obtaining selective drugs for treating infections caused by $L$. donovani, a unicellular parasite causing visceral leishmaniasis, or kala azar, which claims 20,000 to 30,000 deaths per year. Sequence and structural comparisons indicate that the EP is a characteristic which also occurs in the active site of several other important pathogenic protozoa. Therefore, the structure of $L d$ TyrRS could inspire the design of compounds useful for treating several different parasitic diseases. 


\section{Introduction}

The leishmaniases are a variety of diseases caused by more than 20 Leishmania species. These protozoans are transmitted through the bites of infected female phlebotomine sandflies. Approximately 350 million people in the tropics and sub-tropics are at risk of infection, with 0.9 to 1.3 million new cases and 20,000 to 30,000 deaths annually [1,2]. Depending on the species involved, cutaneous, mucocutaneous or visceral leishmaniasis can develop, the latter being the most serious as far as number of deaths is concerned. Caused by L. donovani and L. infantum, visceral leishmaniasis (VL), or kala-azar, is fatal if left untreated in $95 \%$ of the cases. VL is characterized by irregular bouts of fever, weight loss, enlargement of the spleen and liver, and anemia. About 200,000 to 400,000 new cases of VL occur each year and children are the most severely affected group [2,3]. Available drugs used for the treatment of the leishmaniases are mainly pentavalent antimonial complexes, amphotericin $\mathrm{B}$, the aminoglycoside paromomycin, and the alkylphosphocholine miltefosine, either as single or combination treatments $[4,5]$. Most of the available drugs are not oral and, due to their complicated administration regimens, low efficacy for parasite elimination, poor safety profile, occurrence of drug resistance [4] and treatment failure, there is an urgent need to take new oral drug candidates into clinical development [1].

Protein translation is an essential cellular function in Trypanosomatid parasites. The overall fidelity of protein synthesis relies on the accuracy of both codon-anticodon recognition and aminoacyl-tRNA synthesis [6]. Aminoacyl-tRNA synthetases (aaRSs) play a crucial role in accurately pairing each amino acid with its cognate tRNA through a two-step esterification reaction forming aminoacyl-tRNA [7]. The first, ATP dependent, step leads to the formation of an enzyme-bound aminoacyl-adenylate and an inorganic pyrophosphate leaving group. The second step results in the 3'-esterification of the tRNA with the amino acid moiety and generation of AMP as a leaving group, followed by product release [6].

Human cells have two sets of aaRS, one for cytosolic aminoacyl-tRNA synthesis and a second one, of bacterial evolutionary origin, for aminoacylation of mitochondrial tRNAs. In contrast, Trypanosomatids contain only a single set of aaRS genes, with the exception of three amino acids (aspartate, lysine and tryptophan) for which two aaRS genes exist [8]. Hence, in these parasites 17 aaRSs have to function in both subcellular locations. This is a remarkable 
difference between human and parasite aaRS. It suggests that inhibiting any of these aaRS in parasites would have the effect of hindering protein synthesis in the cytosol as well as in the mitochondria.

AaRSs have been recognized as validated antimicrobial drug targets [9-11]. Anti-aaRS compounds can act by blocking the binding site of ATP and/or amino acid, an allosteric site, tRNA recognition, or a secondary editing site [12]. Successes in targeting pathogenic aaRS are encouraging. At least two drugs, mupirocin and tavaborole, are in clinical topical use against, respectively, Staphylococcus aureus infections and onychomycosis. Mupirocin binds both the ATP and Ile binding sites of $S$. aureus isoleucyl-tRNA synthetase (IleRS), while tavaborole $\left(\operatorname{Kerydin}^{\mathrm{TM}}\right.$ ) inhibits fungal leucyl-tRNA (LeuRS) by binding to its editing site [10, 13, 14]. Compounds targeting parasitic protozoan aaRS have been shown to interfere with protozoan cell growth [10, 15]. Also, several natural products inhibit aaRS from important tropical parasites. For instance, Plasmodium falciparum prolyl-tRNA synthetase, lysyl-tRNA synthetase and threonyl-tRNA synthetase are inhibited by the natural products halofuginone, cladosporin and borrelidin, respectively $[12,16,17]$. These compounds have also inhibitory effects on malaria parasite growth in vitro or in vivo [18]. Here we focus on tyrosyl-tRNA synthetase (TyrRS) from L donovani.

TyrRS belongs to the class I aaRSs, characterized by a Rossmann fold and two hallmark sequence motifs ("HIGH" and "KMSKS"). More specifically, TyrRS belongs to subclass Ic, together with tryptophanyl-tRNA synthetase (TrpRS), and contains an "AIDQ" motif characteristic of the ATP binding site. Progress has been made in inhibiting bacterial TyrRSs both with compounds identified from natural sources, as is the case of SB-219383 [19-21], and with synthetized inhibitors [22-25]. However, there have been no significant advances in experimental work with parasitic TyrRSs as drug targets [10]. A structure guided approach in the design of drugs for the treatment of VL would greatly benefit from the knowledge and analysis of new leishmanial TyrRSs structures, in particular from L. donovani and L. infantum.

Recent biochemical and parasitological studies on L. donovani TyrRS (LdTyrRS) have indicated that the enzyme has a cytoplasmic subcellular location and that, as expected, it is essential for cell proliferation. Interestingly, an intriguing additional function, i.e. an effect on inflammation, has been shown in $L$. donovani for secreted TyrRS. The enzyme appears to be involved in attracting neutrophils and binding to macrophages through an N-terminal ELR motif 
triggering further cytokine TNF- $\alpha$ and IL-6 release by host macrophages [26]. Regarding inhibition studies of $L d$ TyrRS, the flavonoid fisetin has shown to have anti-leishmanial properties and its effect has been ascribed to its anti-TyrRS activity [26]. This inhibitory effect of fisetin agrees well with the observation that this compound binds to the active site of L. major TyrRS (LmTyrRS) [27].

In order to provide a structural platform to assist further anti-leishmanial drug development, we report here the $2.75 \AA$ resolution crystal structure of $L d$ TyrRS in complex with the tyrosyl adenylate analog TyrSA (5'-O-[N-(L-tyrosyl)sulfamoyl]adenosine) and a specific anti-LdTyrRS nanobody (NbA) used as crystallization chaperone [28]. Nanobodies are the variable domains of camelid heavy chain-only antibodies which can substantially increase the success of protein crystal growth (28). The structure of $L d T y r R S$ in complex with TyrSA will in particular expand our insight into the binding characteristics of ligands in the neighborhood of the adenine binding pocket, a feature which was not probed in the previous structures of LmTyrRS. Analysis of the structure indicates the presence of promising opportunities to exploit structural differences of the parasite enzyme with both human cytosolic and mitochondrial TyrRS variants. Of particular relevance is the presence of an "extra pocket" (EP) near the adenine binding site in the structure of $L d$ TyrRS. This pocket is absent in the two human homologs and hence provides distinct opportunities to arrive at selective inhibitors. The EP is also present in the structure of L. major TyrRS [27], and, in view of the considerable similarities in the active site regions, is highly likely to occur in other Leishmania species causing human disease. The same holds for TyrRS from related Trypanosomatids, such as Trypanosoma brucei, the causative agent of human African trypanosomiasis, and T. cruzi, responsible for Chagas disease. In addition, TyrRS from the malaria parasite Plasmodium falciparum [29] appears to contain a close variant of the EP. Amino acid sequence comparisons suggest that the EP occurs in even more parasitic protozoa. Hence the EP may allow the development of selective tyrosyltRNA synthetase inhibitors which could become new tools in tackling several major diseases caused by unicellular parasites. 


\section{Materials and methods}

\subsection{LdTyrRS expression and purification}

LdTyrRS was cloned into the AVA0421 vector and expressed in E. coli for subsequent purification. A first round of Ni-NTA affinity chromatography was followed by cleavage of the $\mathrm{N}$-terminal $\mathrm{His}_{6}{ }_{-}$tag using $3 \mathrm{C}$ protease (overnight at $4{ }^{\circ} \mathrm{C}$ ). In a second Ni-NTA step, the cleaved $L d$ TyrRS was purified from the $\mathrm{N}$-terminally $\mathrm{His}_{6}$-tagged 3C protease. A size-exclusion chromatography on a Superdex 200 column (Amersham Pharmacia Biotech) using SEC buffer (25 mM HEPES at pH 7.25, $500 \mathrm{mM} \mathrm{NaCl}, 2 \mathrm{mM}$ TCEP, 5\% glycerol, 0.025\% $\mathrm{NaN}_{3}$ ) was performed and a final yield of $6 \mathrm{mg}$ of pure $L d T \mathrm{TrRS}$ per liter of $E$. coli culture was obtained and concentrated above $15 \mathrm{mg} / \mathrm{mL}$ for co-crystallization with $\mathrm{NbA}$.

\section{2 $\mathrm{NbA}$ production}

$L d$ TyrRS specific nanobodies were generated as previously described [28]. In brief, one llama (Lama glama) was immunized six times with in total $0.9 \mathrm{mg}$ of $L d$ TyrRS. Four days after the final boost, blood was taken to isolate peripheral blood lymphocytes. RNA was purified from these lymphocytes and reverse transcribed by PCR to obtain cDNA. The resulting library was cloned into the phage display vector pMESy4 bearing a C-terminal hexa-His tag and a CaptureSelect sequence tag (Glu-Pro-Glu-Ala). Six different families were selected by biopanning. For this, $L d$ TyrRS was solid phase coated directly on plates, $L d$ TyrRS specific phage were recovered by limited trypsinization. After two rounds of selection, periplasmic extracts were made and subjected to ELISA screens [28].

\section{3 $\mathrm{NbA}$ expression and purification}

$\mathrm{NbA}$ cloned in the pMESy4 vector that carries the pelB sequence coding for the secretion signal peptide of PelB was expressed in E. coli for subsequent purification from the bacterial periplasm. $\mathrm{NbA}$ purification was performed as previously described by Pardon et al. [28]. Briefly, after induction, the bacterial pellet was gently resuspended in TES buffer (200 mM Tris at pH 8.0, 0.5 
$\mathrm{mM}$ EDTA, $500 \mathrm{mM}$ Sucrose) and upon incubation on ice the periplasmic content was recovered by centrifugation. $\mathrm{NbA}$ was purified from the periplasmic content by $\mathrm{Ni}$-NTA affinity chromatography followed by size-exclusion chromatography on a Superdex 75 column (Amersham Pharmacia Biotech) using NbSEC buffer (25 mM HEPES at pH 7.25, $300 \mathrm{mM}$ $\mathrm{NaCl}, 1 \mathrm{mM}$ TCEP, $10 \%$ glycerol, $0.025 \% \mathrm{NaN}_{3}$ ) and concentrated above $10 \mathrm{mg} / \mathrm{mL}$ for cocrystallization with $L d$ TyrRS.

\subsection{Nanobody-LdTyrRS binding studies}

Nanobody $(\mathrm{Nb})$ binding studies were systematically carried out by native gel electrophoresis and size-exclusion chromatography for eight nanobodies. The purified $\mathrm{Nb}$ was first incubated with $L d$ TyrRS for 30 min at $4{ }^{\circ} \mathrm{C}$ and upon a native gel electrophoresis the positions of $L d \mathrm{TyrRS}, \mathrm{Nb}$ and complex were analyzed. The formation of the complex was also tested by comparing the elution peaks on a Superdex 200 column (Amersham Pharmacia Biotech) where LdTyrRS was run as well as the potential $L d \mathrm{TyrRS} \cdot \mathrm{Nb}$ complex formed upon $30 \mathrm{~min}$ incubation at $4{ }^{\circ} \mathrm{C}$.

\section{5 $L d$ TyrRS aminoacylation assay}

The $\mathrm{IC}_{50}$ of TyrSA in the $L d$ TyrRS aminoacylation assay was determined using methods as previously described [30-32]. Briefly, TyrSA (tested in triplicate) was pre-incubated for 15 minutes at room temperature with $0.13 \mathrm{nM} L d$ TyrRS, $500 \mathrm{nM}[3 \mathrm{H}] \mathrm{L}-$ tyrosine $(40 \mathrm{Ci} / \mathrm{mmol}), 0.2$ $\mathrm{mM}$ ATP. $0.1 \mathrm{U} / \mathrm{mL}$ pyrophosphatase, $0.2 \mathrm{mM}$ spermine, $0.2 \mathrm{mg} / \mathrm{mL}$ bovine serum albumin, 2.5 $\mathrm{mM}$ dithiothreitol, $1 \mathrm{mM} \mathrm{MgCl} 2,25 \mathrm{mM} \mathrm{KCl,} 50 \mathrm{mM}$ HEPES-KOH pH 7.6, and 2\% DMSO. The reactions were started with $200 \mu \mathrm{g} / \mathrm{mL}$ tRNA from brewer's yeast (Roche) and incubated for 30 minutes at room temperature without shaking. The reactions were stopped with cold $10 \%$ trichloroacetic acid and processed as previous described [32].

\section{6 $\mathrm{LdTyrRS} \bullet \mathrm{NbA} \cdot \mathrm{Ty}$ YSA complex crystallization}

Purified $L d$ TyrRS and $\mathrm{NbA}$ proteins were incubated on ice for $30 \mathrm{~min}$ at a 1:2 molar ratio followed by buffer exchange to crystallization buffer (25 mM HEPES at pH 7.25, $100 \mathrm{mM} \mathrm{NaCl}$, $1 \mathrm{mM}$ TCEP-HCl, $5 \%$ glycerol, $\left.0.025 \% \mathrm{NaN}_{3}\right)$. The complex $(5 \mathrm{mg} / \mathrm{mL})$ was then incubated with $200 \mu \mathrm{M}$ of TyrSA (5'-O-[N-(L-tyrosyl)sulfamoyl]adenosine) on ice for 30 - 60 min prior to setting up the crystallization tray. The crystals were obtained after 5 - 7 days at room temperature 
by vapor diffusion using sitting drops equilibrated against a reservoir containing $0.1 \mathrm{M}$ sodium cacodylate $\mathrm{pH} 5.7,22 \%$ PEG 4,000. The drops contained $1 \mu \mathrm{L}$ of $L d \mathrm{TyrRS} \cdot \mathrm{NbA} \cdot \mathrm{TyrSA}$ complex at $5 \mathrm{mg} / \mathrm{mL}$ and $1 \mu \mathrm{L}$ of reservoir solution. After growth, crystals were flash frozen in liquid nitrogen in cryo-solution (25\% glycerol in reservoir solution) and stored until data collection.

\subsection{Data Collection and Structure Determination}

Data was collected under cryogenic conditions at the Stanford Synchrotron Radiation Lightsource (SSRL) using beamline 12-2 at a wavelength of $1 \AA$. Data processing was carried out with the program HKL2000 [33]. Initial phases were obtained by molecular replacement using Phaser [34] with as models the structure of L. major TyrRS•Tyrosinol ([27]; PDB: 3P0J) and a NbA homology model generated by Phyre2 [35]. This was followed by iterations of manual building and rebuilding using Coot [36] alternated with refinement of the structure with REFMAC5 [37]. Refinement restraints for TyrSA were obtained with the Grade web server [38]. The structure validation server MolProbity [39] was used throughout the process for structure validation. The final data collection and crystallographic refinement statistics are given in Table 1. Pymol [40] was used to create the figures. Coordinates and structure factors of the LdTyrRS $\bullet \mathrm{NbA} \bullet$ TyrSA complex have been deposited in the Protein Data Bank under the PDB ID: 5USF. 


\section{Results}

\subsection{Leishmania donovani tyrosyl-tRNA synthetase structure}

The crystal structure of the $L d \mathrm{TyrRS} \bullet \mathrm{NbA} \bullet$ TyrSA complex was determined at a resolution of $2.75 \AA$ (Table 1), where $\mathrm{NbA}$ is a nanobody to be described below, TyrSA a tyrosyl adenylate analog, and the symbol "•" indicates a non-covalent complex. The crystals contain two copies of the $L d$ TyrRS $\bullet \mathrm{NbA} \bullet$ TyrSA complex in the asymmetric unit (ASU). Each $L d$ TyrRS molecule is bound to one $\mathrm{NbA}$ molecule (Figure 1). Canonical TyrRSs are formed by two identical monomers, each consisting of a catalytic domain (CD) and an anticodon binding domain (ABD). Instead, the $75 \mathrm{kDa} L d$ TyrRS chain is a pseudo-dimer where the structurally similar N- and Cterminal pseudo-monomers share only $23 \%$ sequence identity. As described for LmTyrRS [27], the $\mathrm{N}$ - and C-terminal pseudo-monomers are connected by a flexible linker between $\alpha 14$ and $\beta 9$ (Figure 1A). The N-terminal pseudo-monomer of $L d$ TyrRS contains the three motifs characteristic for the catalytic domain of Class I tRNA synthetases: "HIGH" $\left({ }^{46} \mathrm{HIAQ}^{49}\right.$ in LdTyrRS), "AIDQ" ( ${ }^{182} \mathrm{GLDQ}^{185}$ in LdTyrRS) and "KMSKS" $\left({ }^{222} \mathrm{KMSKS}^{226}\right.$ in $\left.L d \mathrm{TyrRS}\right)$ (Figure $1 \mathrm{~B}$ ). These motifs have been shown to be involved in the catalytic activity of TyrRS enzymes [27, 41]. The motifs are absent in the C-terminal pseudo-monomer, suggesting that this half of the molecule is not able to perform amino acid activation. This is in agreement with the observations that TyrSA is bound to the active site in the N-terminal CD of $L d$ TyrRS, but not to the C-terminal CD. Hence, the N-terminal CD is called the "functional CD" and the C-terminal $\mathrm{CD}$ the "non-functional CD" (Figure $1 \mathrm{~A}$ ).

The opposite is true for the ABD. Although both ABDs contain the sequence motifs "AC1" $\left({ }^{244} \mathrm{KIRQAYC}{ }^{250}\right.$ and ${ }^{578} \mathrm{KIKKAYS}^{584}$ in $\left.L d \mathrm{TyrRS}\right)$ and "AC2" $\left({ }^{313} \mathrm{VSEDALK}^{319}\right.$ and ${ }^{636}$ LHPADLK $^{642}$ in LdTyrRS) involved in the recognition of the tRNA anticodon arm, as described for LmTyrRS [27], the loop located between $\beta 7$ and $\beta 8$ in the N-terminal ABD is considerably shorter than the corresponding loop between $\beta 14$ and $\beta 15$ in the C-terminal pseudomonomer. This loop is responsible for binding the anticodon base G34 of tRNA ${ }^{T y r}$ according to the structure of Saccharomyces cerevisiae TyrRS [42]. The short loop in the N-terminal ABD homolog is unable to engage with this base (Figure $1 \mathrm{C}$ ). Therefore, the N-terminal ABD is called the "non-functional ABD" and the C-terminal ABD the "functional ABD" (Figure $1 \mathrm{~A}$, C). 
Table 1. Crystallographic data collection and refinement statistics.

\begin{tabular}{|c|c|}
\hline Parameters & $\begin{array}{c}L d \mathrm{TyrRS} \bullet \mathrm{NbA} \bullet \mathrm{TyrSA} \\
(\mathrm{PDB}: 5 \mathrm{USF})\end{array}$ \\
\hline $\begin{array}{l}\text { Data collection } \\
\text { Space group } \\
\text { Cell dimensions: } a, b, c(\AA) \\
\text { Resolution }(\AA) \\
\\
R_{\text {merge }} \\
R_{\text {pim }} \\
\text { Observed reflections } \\
\text { Unique reflections } \\
\text { Mean } I / \sigma I \\
\text { Multiplicity } \\
\text { Completeness }(\%) \\
C C_{1 / 2}\end{array}$ & $\begin{array}{c}P 6_{5} \\
96.18,96.18,351.83 \\
83.29-2.75 \\
(2.85-2.75) \\
0.210(1.911) \\
0.069(0.659) \\
488525(43134) \\
47547(4632) \\
8.7(2.0) \\
10.3(9.3) \\
100.0(100.0) \\
0.995(0.607)\end{array}$ \\
\hline $\begin{array}{l}\text { Refinement } \\
\text { Resolution }(\AA) \\
\text { Reflections used } \\
R_{\text {work }} / R_{\text {free }} \\
\text { Number of atoms }\end{array}$ & $\begin{array}{c}83.29-2.75 \\
45156 \\
0.19 / 0.24\end{array}$ \\
\hline $\begin{array}{l}\text { Protein } \\
\text { TyrSA } \\
\text { Water } \\
\text { Number of residues }\end{array}$ & $\begin{array}{l}12283 \\
70 \\
353 \\
1600\end{array}$ \\
\hline $\begin{array}{l}\text { Average } B \text {-factors }\left(\AA^{2}\right) \\
\text { Protein } \\
\text { TyrSA } \\
\text { Water }\end{array}$ & $\begin{array}{l}66.0 \\
49.8 \\
55.9\end{array}$ \\
\hline $\begin{array}{l}\text { R.m.s. deviations } \\
\text { Bond lengths }(\AA) \\
\text { Bond angles }\left({ }^{\circ}\right)\end{array}$ & $\begin{array}{l}0.01 \\
1.44\end{array}$ \\
\hline $\begin{array}{l}\text { Ramachandran plot } \\
\text { Favored (\%) } \\
\text { Outlier (\%) }\end{array}$ & $\begin{array}{c}97 \\
0\end{array}$ \\
\hline $\begin{array}{l}\text { Ligand (TyrSA) } \\
\text { Average LLDF }^{@} \\
\text { Average RSR }^{\wedge}\end{array}$ & $\begin{array}{c}-0.53 \\
0.14\end{array}$ \\
\hline
\end{tabular}

Values in parentheses are for the highest-resolution shell.

"Ramachandran Plot statistics as reported by the wwPDB validation report

${ }^{\circledR}$ Local ligand density fit as reported by the wwPDB validation report

${ }^{\wedge}$ Real space $\mathrm{R}$ value as reported by the wwPDB validation report 


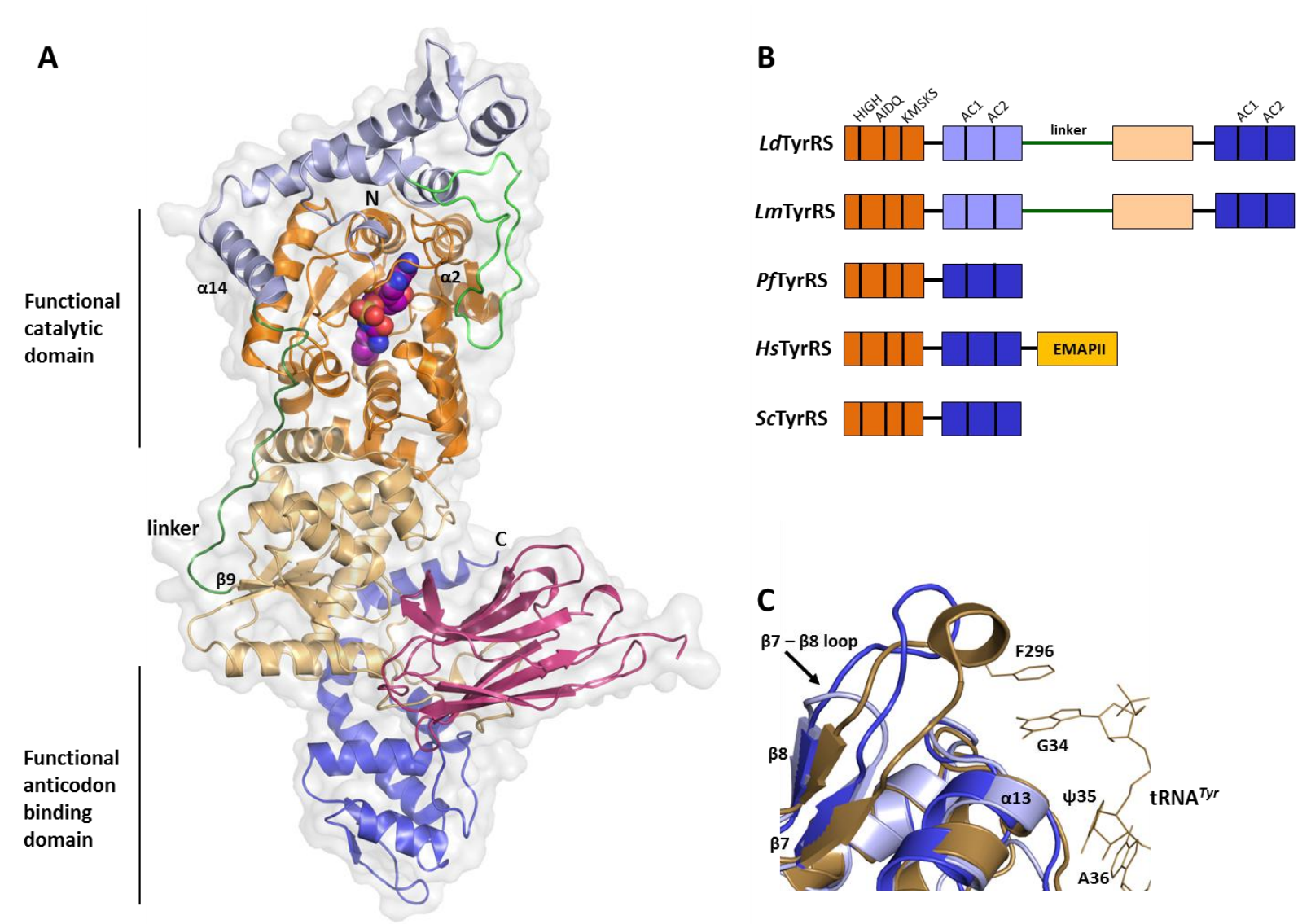

Figure 1. Domain organization of pseudo-dimeric LdTyrRS. A) The asymmetric LdTyrRS pseudo-dimer structure in complex with nanobody A (pink) and the tyrosyl adenylate analog TyrSA (CPK model). The N-terminal functional catalytic domain (CD) is shown in dark orange, the $\mathrm{C}$-terminal nonfunctional $\mathrm{CD}$ in light orange; the $\mathrm{N}$ terminal non-functional ABD in light blue, the C-terminal functional ABD in dark blue. The linker connecting the $\mathrm{N}$-terminal and C-terminal pseudo-monomers of $L d$ TyrRS is shown in dark green, while the plant/plastid insertion present in Trypanosomatid TyrRSs, described previously by Larson et al. [27], in the N-terminal ABD is shown in bright green. The ELR motif is located in the exposed $\alpha 2$ in the functional CD. B) Domain organization of TyrRS from L. donovani, L. major, P. falciparum (PfTyrRS), human (HsTyrRS) and the yeast S. cerevisiae (ScTyrRS). Domain colors correspond to the ones in LdTyrRS structure in part A. The characteristic motifs HIGH, AIDQ and KMSKS in the catalytic domain as well as AC1 and AC2 in the anticodon binding domains are depicted. When processed by an elastase enzyme HsTyrRS gives rise to an N-terminal TyrRS known as Mini TyrRS and a Cterminal EMAPII-like domain (yellow), which has cell signaling activity [29]. (EMAP stands for: "Endothelial Monocyte-Activating Polypeptide II"). C) Anticodon recognition regions of the N-terminal and C-terminal pseudomonomers of LdTyrRS and ScTyrRS. The LdTyrRS N-terminal (light blue) and C-terminal (dark blue) ABDs are shown superimposed with the $S c$ TyrRS $\bullet$ RNA $^{T y r}$ complex (PDB ID: 2dlc) (sand). Residue F296, implied in $S c$ TyrRS tRNA $^{T y r}$ recognition, and the tRNA ${ }^{T y r}$ anticodon bases guanine - pseudo-uridine - adenine $\left({ }^{34} \mathrm{G}-\psi-\mathrm{A}^{36}\right)$ are depicted. The secondary structure elements in the LdTyrRS N-terminal ABD are labeled. A black arrow points to the shortened loop between $\beta 7$ and $\beta 8$ in the N-terminal ABD. 
The superposition of the two $L d$ TyrRS chains A and B in the $L d$ TyrRS $\bullet N b A \cdot T y r S A$ crystals yields an overall r.m.s.d of $1.57 \AA$ for $678 \mathrm{C}^{\alpha}$ atoms. All four individual domains of the two chains in the asymmetric unit are highly similar, with r.m.s.d values ranging between 0.25 and $0.77 \AA$. The $\mathrm{N}$ - or $\mathrm{C}$ - terminal pseudo-monomers of the $\mathrm{A}$ and $\mathrm{B}$ chains are also similar to each other, with r.m.s.d. values of $0.52 \AA$ and $0.77 \AA$ after superposition. However, after applying the superposition operation of the N-terminal pseudo-monomers to the entire chains, there appears to be a change in orientation of the two C-terminal pseudo-monomers, resulting in a $10.4 \AA$ displacement at the farthest end of the C-terminal pseudo-monomer loop between $\beta 14$ and $\beta 15$ (Figure 2). These comparisons of chains $A$ and $B$ indicate that there is intrinsic flexibility within the $L d$ TyrRS molecule, in particular between the N- and C-terminal pseudomonomers. 


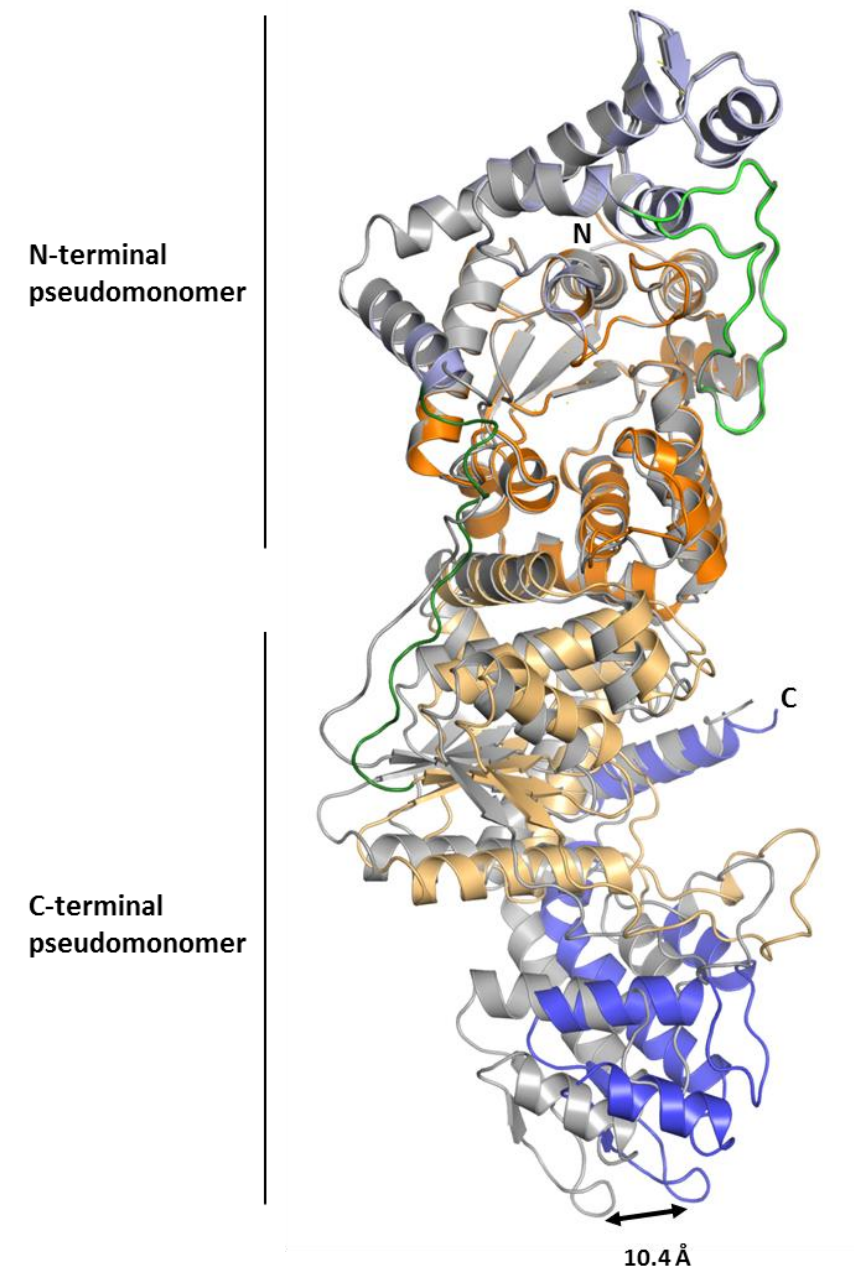

Figure 2. Analysis of $L d T y r R S$ flexibility through superposition of $L d T y r R S$ chains A and B within the crystal. Chain A is colored by domains as described in Figure 1, while chain B is shown in grey. When superimposing the N-terminal pseudo-monomers of both $L d$ TyrRS copies in the crystal, a shift in the C-terminal half of the enzyme is made evident especially in the farthest end of the C-terminal pseudo-monomer where a $10.4 \AA$ displacement occurs (black arrow). 
When considering the $L d$ TyrRS active site, all chain A residues are well defined, while in chain B density for two residues $\left(\mathrm{S}^{224}\right.$ and $\mathrm{K}^{225}$ ) belonging to the ${ }^{221} \mathrm{KMSKS}^{226}$ loop is missing. Therefore we focus our structural analysis on chain A of $L d$ TyrRS. The KMSKS loop adopts a closed conformation in our $L d$ TyrRS structure with the tyrosyl adenylate analog TyrSA bound (Figure 3). Interestingly, the KMSKS loop was also found in a closed conformation in LmTyrRS, even in the absence of ATP or tyrosyl adenylate analogs [27].

Not only the KMSKS loop can have an open and closed conformation, also the entire active site can be in an open or closed state. These two distinct conformational states have been described by Larson et al. [27] for the active site in the LmTyrRS•Tyrosinol and $L m$ TyrRS $•$ Fisetin crystal structures. In the functional catalytic domains of $L d$ TyrRS $\bullet$ TyrSA both chains adopt the closed state (Figure 3). In this state, the loop containing residues 146-154 is well-ordered and reaches the proximity of the sulfamoyl group of TyrSA, where the phosphate of tyrosyl adenylate or the $\alpha$ and $\beta$ phosphates of ATP would be during the catalytic reaction by TyrRS. As in the LmTyrRS closed state, the $L d$ TyrRS $\alpha 4$ and the loop connecting this helix with $\alpha 5$ curl over the active site to interact with residues $40-43$ preceding the HIGH motif $\left({ }^{47} \mathrm{HIAQ}^{49}\right.$ in $L d$ TyrRS) involved in the enzyme's catalytic activity (Figure 3). 


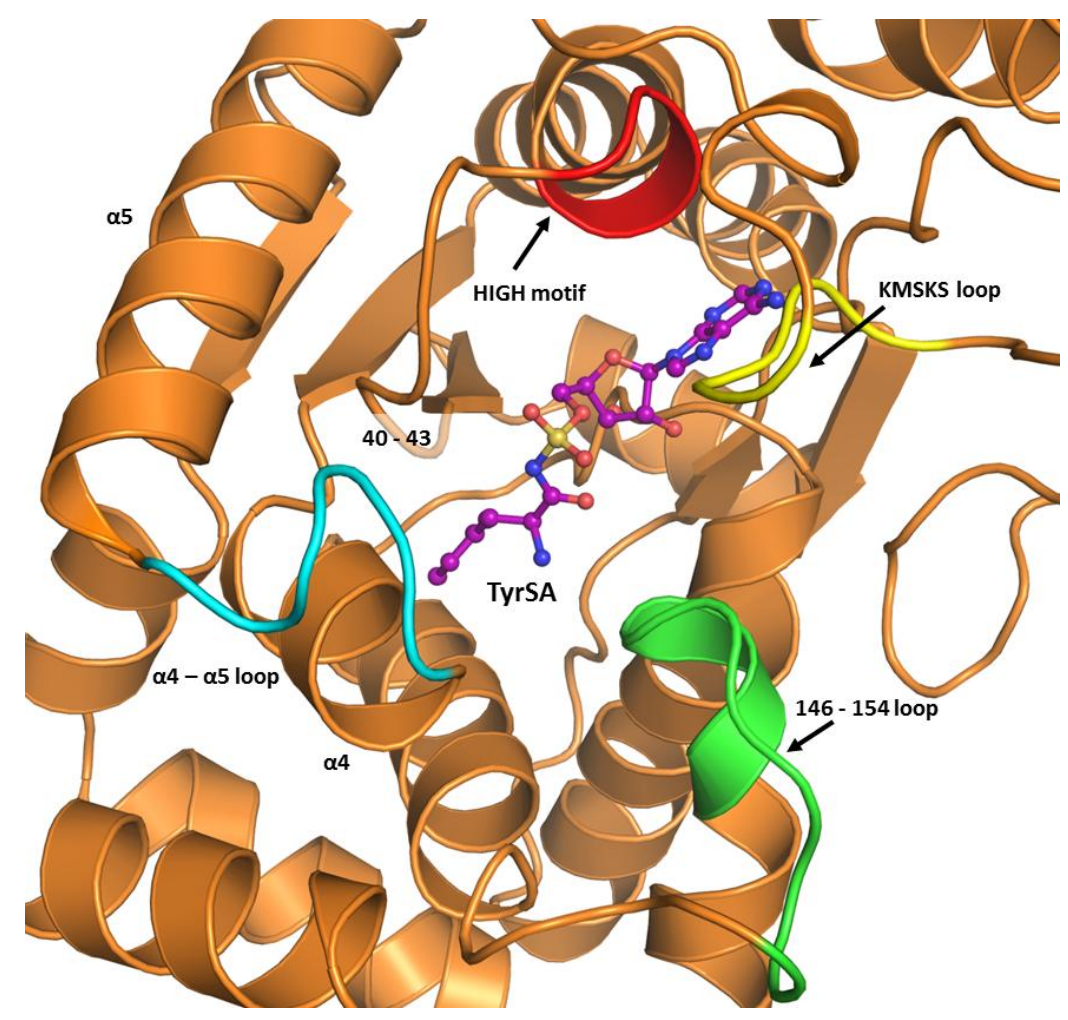

Figure 3. Closed conformational state of the active site in the $L d$ TyrRS $\bullet$ TyrSA structure. $L d$ TyrRS in orange while TyrSA in purple. The various loops and motifs that are well ordered in a closed conformation of the enzyme's active site are colored as follows: HIGH motif in red, KMSKS loop yellow, $\alpha 4-\alpha 5$ loop cyan, and 146-154 loop green. 


\section{2 $\mathrm{NbA}$ structure and its interactions with $L d \mathrm{TyrRS}$}

After various failed attempts to crystallize $L d$ TyrRS, we hypothesized that some flexibility in the four domain pseudo-dimer was impairing crystal formation. With the use of nanobodies as crystallization chaperones we aimed to freeze $L d$ TyrRS in a conformation more amenable for crystal growth, while the nanobody might also be able to establish favorable crystal contacts. Nanobodies are small compact single-domain fragments of the original heavy-chain camelid antibodies that retain their full antigen-binding capacity. Typically three variable loops of $\beta$ strands, referred to as the complementarity determining regions (CDRs), are responsible for binding to the antigen. The usually long CDR3 loop of nanobodies can have a special significance since cryptic epitopes located in cavities or clefts of the antigenic protein are sometimes recognized by it [28]. However, in the $L d \mathrm{TyrRS} \bullet \mathrm{NbA} \bullet$ TyrSA complex the CDR3 loop in anti-LdTyrRS llama nanobody A (NbA) does not interact extensively with $L d$ TyrRS as described below.

We tested 4 anti- $L d$ TyrRS nanobodies as crystallization chaperones before obtaining high quality $L d$ TyrRS crystals with $\mathrm{NbA}$. The presence of the tyrosyl adenylate analog TyrSA was essential as well, and its interaction with the enzyme will be described later. The 1:1 $L d$ TyrRS $\bullet N b A$ complex was purified by size exclusion chromatography (SEC) and the binding of $\mathrm{NbA}$ to $L d \mathrm{TyrRS}$ was verified through the shift of the $280 \mathrm{~nm}$ absorbance elution peak (Figure $4 \mathrm{~A}$ ), corroborating the formation of a larger molecular weight species. The presence of both proteins $L d$ TyrRS and NbA in the larger molecular weight species was confirmed by SDS-PAGE (Figure $4 \mathrm{~B}$ ). In addition, native gel binding assays were performed supporting the interaction between the two proteins (not shown).

$\mathrm{NbA}$ is a $14 \mathrm{kDa}$ protein and consists of two $\beta$-sheets with a Greek key topology (Figure $4 \mathrm{C}$ ) held together by a disulfide bond (C22-C96). Interestingly, the CDR3 loop in NbA adopts an anti-parallel pair of $\beta$-strands connected by a short loop of 4 residues. Side chains of the $\beta$ strands of CDR3 do not interact with $L d$ TyrRS, but the short connecting loop does. In this case, CDR2 buries a larger solvent accessible surface area $\left(511 \AA^{2}\right)$ when interacting with $L d$ TyrRS than CDR3 (377 $\AA^{2}$ ) (calculated by PISA [43]).

With a buried surface area (BSA) of $1947 \AA^{2}$, one NbA molecule interacts mainly with the non-functional $\mathrm{CD}$ of each $L d$ TyrRS chain in the crystal, and makes a few additional contacts 
with the functional ABD. The corresponding estimated free energy of dissociation $\Delta^{i} G$ is -8 $\mathrm{kcal} / \mathrm{mol}$ (calculated by PISA). The contacts between the variable region of NbA and $L d \mathrm{TyrRS}$ are predominantly hydrophilic (Figure 4 D). Residues N31 and W33 of CDR1, residues R50 and G54 of CDR2, and, residue R102 of CDR3 (through a water molecule), make hydrogen bonds with $L d$ TyrRS. Side chain carbon atoms of residues N56 (CDR2) and Y103 (CDR3) are engaged in hydrophobic interactions with the enzyme. In addition, several frame work residues, not located in CDR loops, establish polar (R19, S69 and S71) and hydrophobic (Y80) interactions with the synthetase.

It is of interest to see if $\mathrm{NbA}$ has a stabilizing effect on $L d$ TyrRS, thereby promoting possibly crystal growth. The comparison with the LmTyrRS structure is helpful here. The loop 541-571 connecting the non-functional CD with the functional ABD in LdTyrRS exhibits a welldefined density, but the equivalent loop in the three LmTyrRS crystal structures available is half or completely disordered [27]. Hence, it seems reasonable to conclude that $\mathrm{NbA}$ diminishes the flexibility of an exposed loop in $L d$ TyrRS.

A second reason why nanobodies can promote crystal growth is by generating additional crystal contacts. In the $L d \mathrm{TyrRS} \cdot \mathrm{NbA} \bullet T y r S A$ complex, $\mathrm{NbA}$ bound to chain A establishes crystallographic interactions with the other $L d$ TyrRS copy (Chain B) with a BSA of $792 \AA^{2}$. Moreover, NbA also contacts a symmetry-related NbA copy with a BSA of $969 \AA^{2}$. The nanobody is clearly engaged in extensive interactions with various protein chains in the crystal. Most likely $\mathrm{NbA}$ increases the probability of crystal growth by making numerous crystal contacts and by diminishing the flexibility of an exposed loop. 

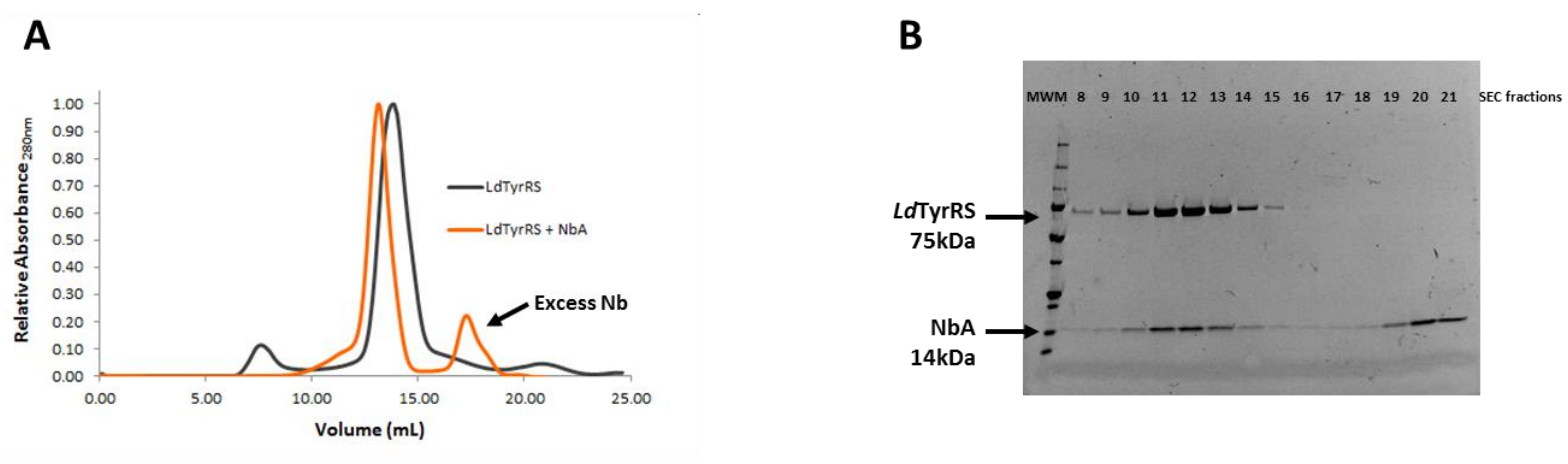

C

D
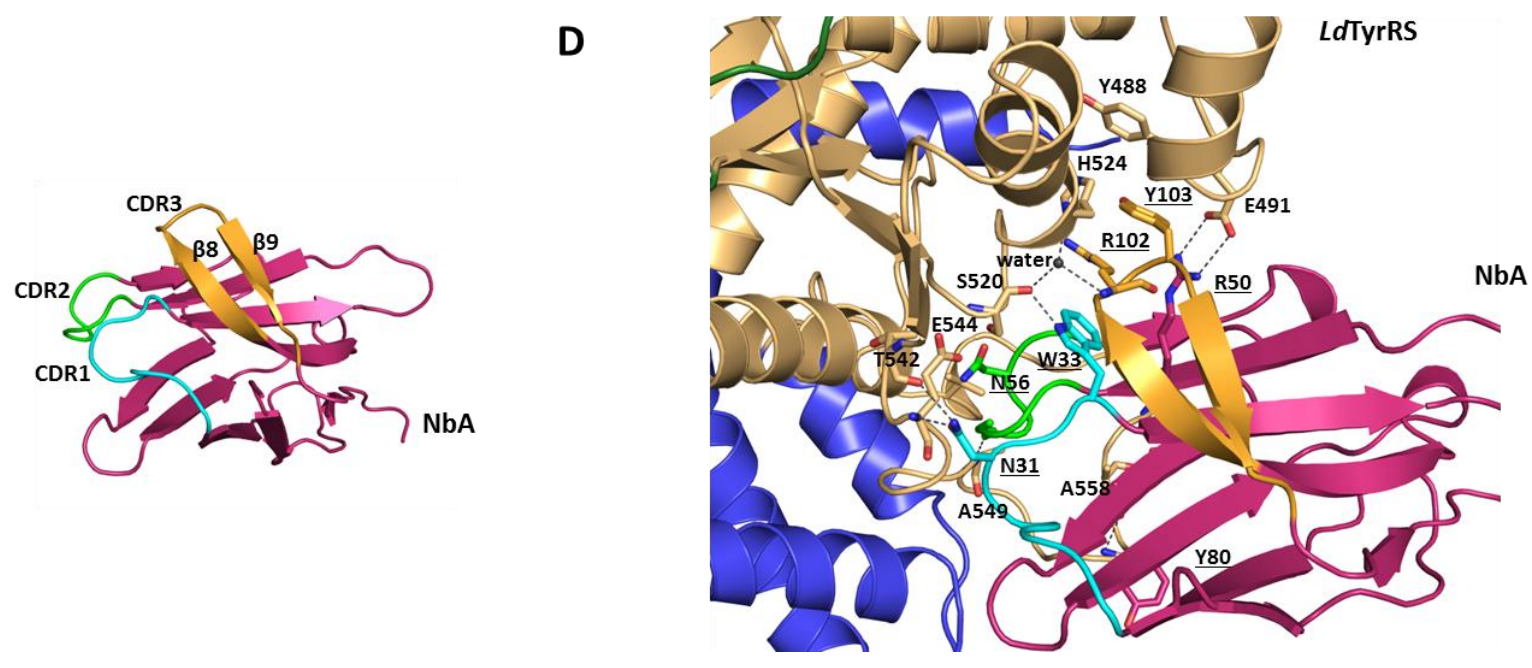

Figure 4. Interactions between $L d$ TyrRS and NbA. A) SEC elution profile of purified $L d$ TyrRS (grey) and $L d$ TyrRS $\bullet N b$ A complex (orange). B) SEC fractions were run in SDS-PAGE to corroborate the formation of the 1:1 complex. The low molecular weight present in the SEC chromatogram was proven to be excess NbA present in the mix $L d$ TyrRS/NbA that was loaded into the SEC column. C) NbA structure (pink) showing the three CDR loops: CDR1 (green), CDR2 (cyan) and CDR3 (gold). D) $L d$ TyrRS $\bullet N b A$ complex interactions. The underlined residues correspond to $\mathrm{NbA}$. Most of the interactions are polar (doted lines). The three CDR loops are involved in interactions with the enzyme (in light orange). 


\subsection{TyrSA binds to $L d$ TyrRS with its adenine ring near an extra pocket (EP)}

The tyrosyl adenylate analog TyrSA (5'-O-[N-(L-tyrosyl)sulfamoyl]adenosine) (Figure $5 \mathrm{~A}$ ) was necessary for obtaining well diffracting $L d$ TyrRS crystals. As measured by ATP depletion assays, TyrSA binds tightly to $L d$ TyrRS with an $\mathrm{IC}_{50}$ of $0.69 \mathrm{nM}$. The inhibitor was found to occupy the active site of the functional CD (Figure $5 \mathrm{~B}$ ) in both $L d$ TyrRS chains in the asymmetric unit. The high affinity of the compound for the enzyme is most probably explained by the large number of interactions with $L d$ TyrRS active site residues, limiting thereby the flexibility of this region. This decrease in motility is likely responsible for the fact that TyrSA was crucial for obtaining well diffracting crystals of the $L d \mathrm{TyrRS} \cdot \mathrm{NbA}$ complex.

The $L d$ TyrRS active site contains two critical pockets: the tyrosine binding pocket (YBP) where the tyrosyl group of TyrSA is situated, and the adenine binding pocket (ABP) where the adenine moiety of TyrSA binds (Figure $5 \mathrm{C}$ ). As evidenced through the use of PoseView as part of the ProteinPlus structure-based modelling software tools [44, 45], the tyrosyl adenylate analog contacts many $L d$ TyrRS active site residues (BSA of $933 \AA^{2}$ ), mainly through hydrogen bonds, although a few hydrophobic interactions are present as well. Residues making hydrogen bonds with the TyrSA tyrosyl group in the YBP are Y36, Y163, Q167, D170 and Q185. Residues G38, A72 and F75 are responsible for the hydrophobic interactions between enzyme and tyrosine moiety in the YBP.

A hydrogen bond is established between an oxygen atom belonging to the sulfamoyl group of TyrSA and the main chain nitrogen atom of residue E40 of LdTyrRS. The residues responsible for hydrogen bond interactions with the ribose ring are D37, G182 and D184. In the ABP, hydrogen bonds with the adenine moiety are made by H210 and L213, while M212 makes hydrophobic contacts with the adenine ring. 
A
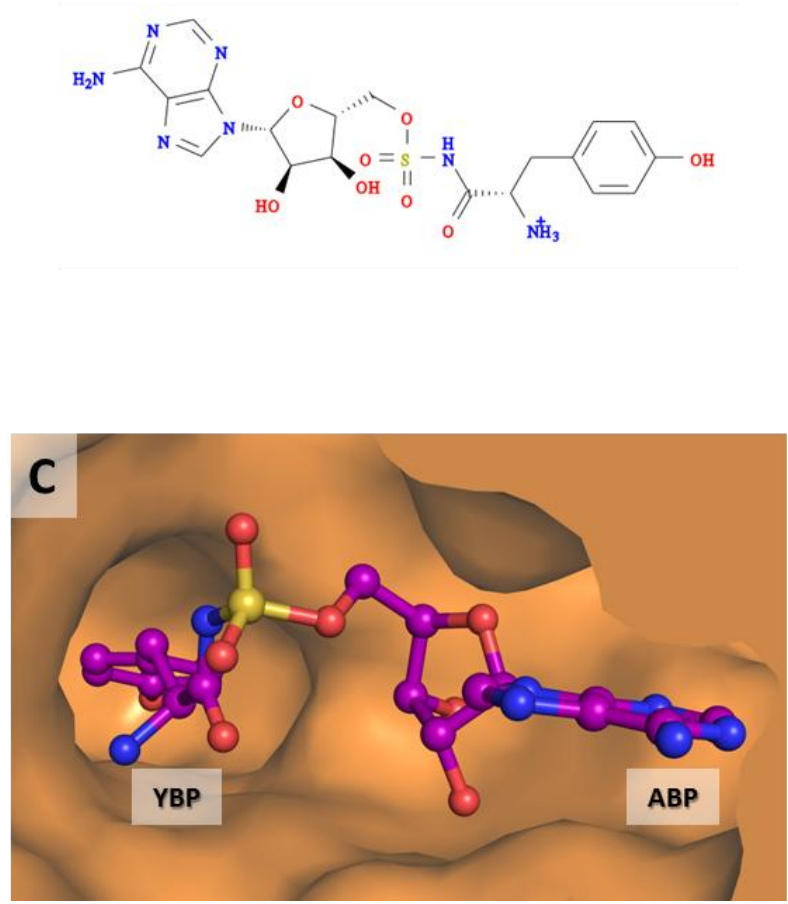
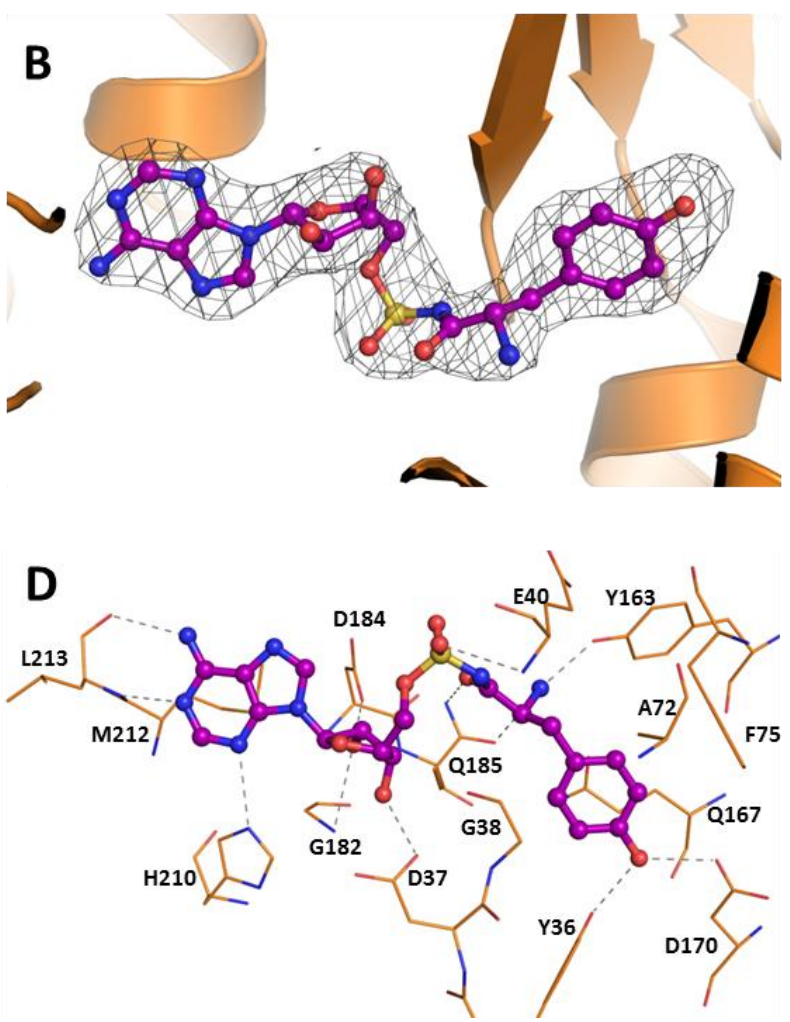

Figure 5. TyrSA binding to $L d$ TyrRS. A) Chemical structure of TyrSA. B) $L d$ TyrRS $\bullet N b A \bullet T y r S A$ with difference electron density map calculated by omitting TyrSA, contoured at $3 \sigma$ (positive density in grey, negative density in red). C) General features of TyrSA binding mode. The protein surface and the two pockets, tyrosine binging pocket (YBP) and adenine binding pocket (ABP), where the compound is bound are shown. D) Extensive hydrogen bond network in the $L d$ TyrRS/TyrSA interaction.

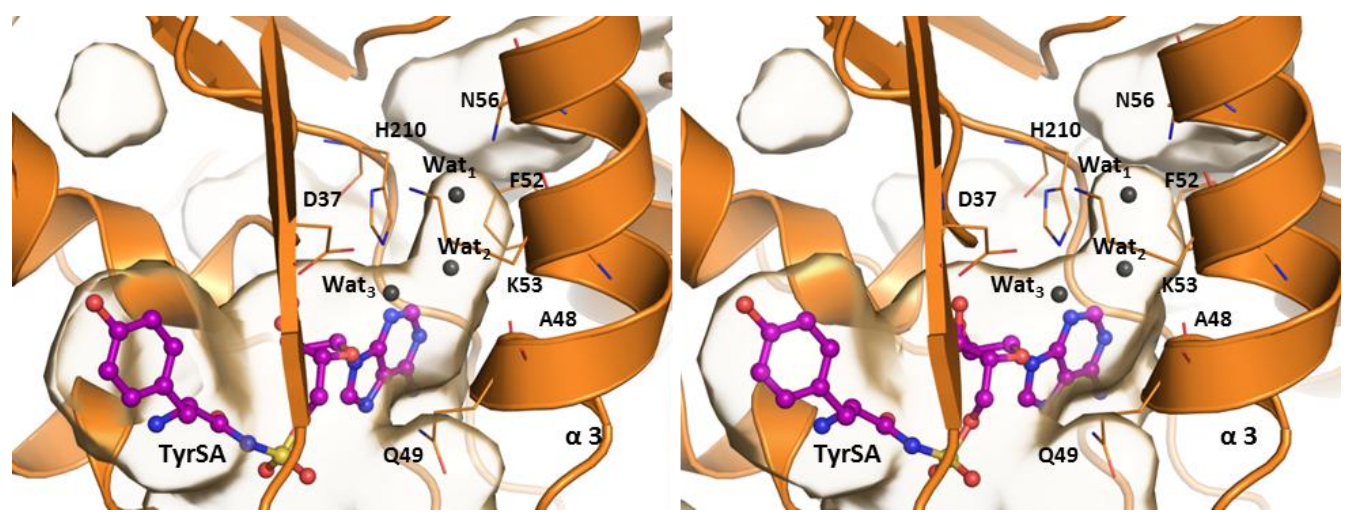

Figure 6. $L d$ TyrRS residues forming the extra pocket (EP). Stereo view of the $L d$ TyrRS catalytic site indicating the residues creating the EP. Most of the residues involved in the EP formation are part of helix $\alpha 3$, in addition to residues D37 and H210. TyrSA molecule in purple. Three water molecules, Wat1, Wat2 and Wat3 are located in the pocket and drawn as grey spheres. 
Further analysis of the active site near the ABP showed a previously not reported feature: a pocket in $L d$ TyrRS close to the TyrSA adenine moiety (Figure 6). This pocket, called "extra pocket" (EP), which is also present in the L. major TyrRS structure, is mainly lined by residues belonging to helix $\alpha 3$ as well as by the side chain of residue H210. Helix $\alpha 3$ atoms contributing to the pocket (Figure 6) are the carbonyl oxygen of A48, and side chain atoms of Q49, F52, K53 and N56. The EP of $L d$ TyrRS harbors two deeply buried water molecules. The most buried water molecule (Wat1) makes hydrogen bonds with the side chains of residues K53 and N56 and with Wat2. Wat2 engages in hydrogen bonding with the main chain carbonyl oxygen of A48 and with Wat 1 and Wat3. The third water molecule (Wat3) interacts with (i) the ether oxygen atom of the TyrSA ribose ring, (ii) Wat2 in the EP, and (iii) the carboxylate group of residue D37 at the pocket entrance. The observation of this extra pocket (EP) in $L d$ TyrRS and $L m$ TyrRS turned out to be particularly interesting when comparing the TyrRS enzymes from the human host and a range of unicellular parasites.

\subsection{The extra pocket (EP) of $L d$ TyrRS is absent in the human TyrRS enzymes}

In order to discover new opportunities for drug design, the active sites of $L d$ TyrRS and the two HsTyrRS enzymes were compared (Figure 7; for a list of TyrRS structures compared with LdTyrRS in this paper see Table 2). While the YBPs are highly similar among the compared TyrRS structures, the analysis of the ABP reveals interesting amino acid differences between human and parasite enzymes as also described by Larson et al. [27] on the basis of the comparison of their $L$. major TyrRS structure with the two human TyrRS enzymes. The basic conclusions of these authors are confirmed by our current $L d$ TyrRS structure.

Interestingly, the EP offers additional exploitable differences between the enzymes from parasite and host. When superimposing the L. donovani and human TyrRS structures, a substantial difference in position of $\alpha 3$ in the human enzymes $v s$. parasite enzyme is evident (Figures 7 and 8). Specifically, in cytosolic $H s$ TyrRS, the helix corresponding to the $L d$ TyrRS $\alpha 3$ helix is moved $3.0 \AA$ towards the EP of $L d$ TyrRS (Figure $8 \mathrm{~B}$ ), while in the mitochondrial HsTyrRS structure this helix is moved by $4.6 \AA$ towards the EP of $L d$ TyrRS (Figure 8 C, D). As result of this shift, the EP is absent in the human enzymes. 
Table 2. Compared structures of tyrosyl tRNA synthetases.

\begin{tabular}{|c|c|c|c|c|}
\hline PDB ID & Organism & Ligand(s) & Crystallized TyrRS & Reference \\
\hline 5 usf & Leishmania donovani & TyrSA and nanobody & Full length & This publication \\
\hline $3 \mathrm{p} 0 \mathrm{~h}$ & Leishmania major & Fisetin & Full length & [27] \\
\hline $3 \mathrm{p} 0 \mathrm{j}$ & Leishmania major & Tyrosinol & Full length & [27] \\
\hline $3 \mathrm{vgj}$ & Plasmodium falciparum & TyrAMP & Full length & [29] \\
\hline $4 q b t$ & Homo sapiens Cytosolic & L-Tyrosine & Truncated (1-341) & [46] \\
\hline 2pid & Homo sapiens Mitochondrial & TyrSA & Full length & [47] \\
\hline $2 \mathrm{dlc}$ & Saccharomyces cerevisiae & Tyr-AMP analogue and tRNA ${ }^{\mathrm{Tyr}}$ & Truncated (1-364) & [42] \\
\hline 1jii & Staphylococcus aureus & SB-219383 & Full length & [20] \\
\hline $2 \mathrm{jan}$ & Mycobacterium tuberculosis & None & Full length & [48] \\
\hline $1 \mathrm{vbm}$ & Escherichia coli & TyrSA & Truncated (1-322) & [41] \\
\hline $1 \mathrm{j} 1 \mathrm{u}$ & Methanocaldococcus jannaschii & L-Tyrosine and tRNA $^{\text {Tyr }}$ & Full length & [49] \\
\hline
\end{tabular}




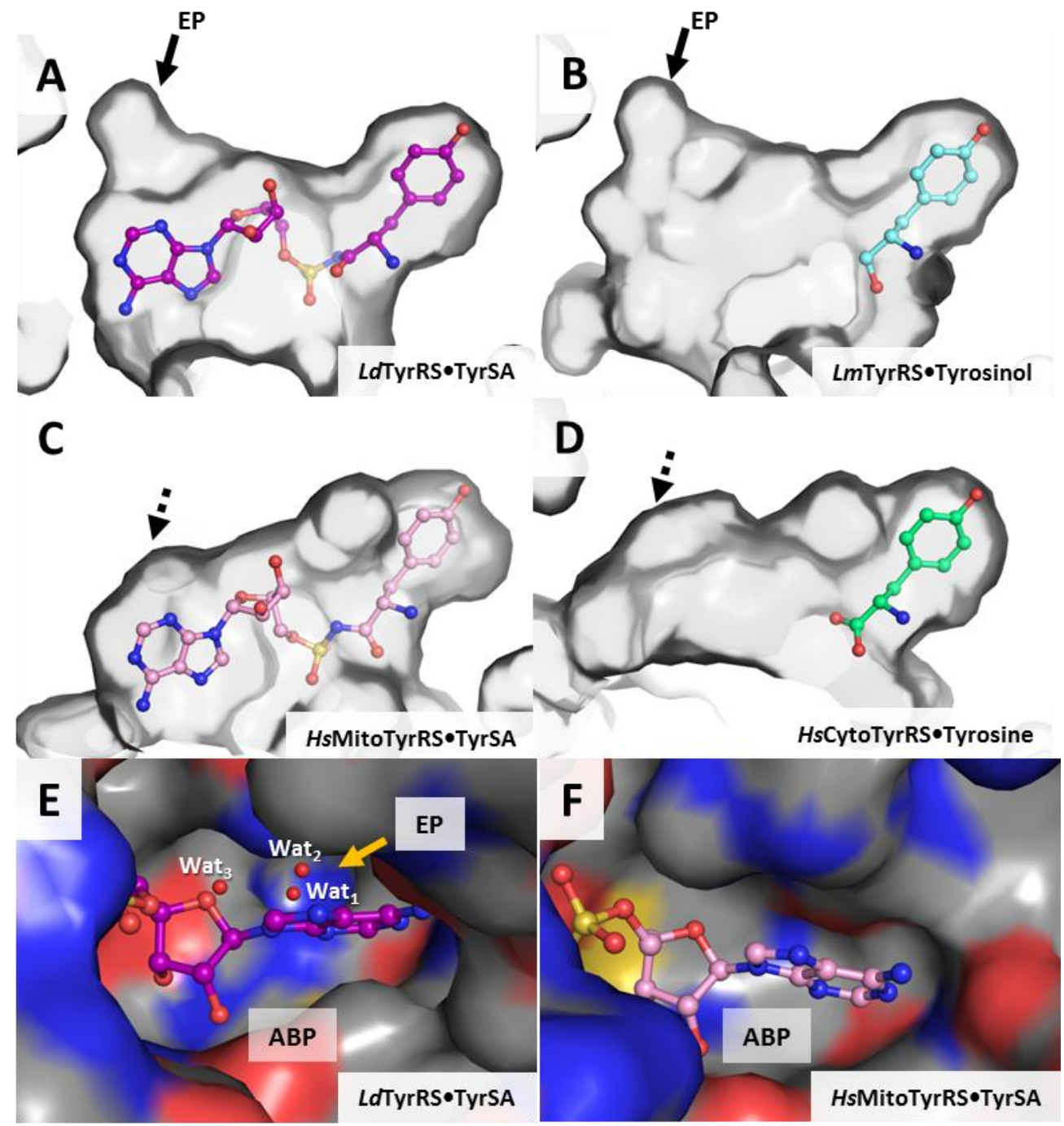

Figure 7. LdTyrRS catalytic pocket surface representation and comparison to human TyrRSs. In panels A, B and E, the extra pocket (EP) is labeled. A) The LdTyrRS•TyrSA structure in complex with TyrSA with purple carbon atoms. B) The LmTyrRS $\bullet$ Tyrosinol structure with the tyrosinol molecule with cyan carbon atoms (PDB: 3POJ). The EP in the L. major enzyme is very similar to that in L. donovani TyrRS. C) HsMitoTyrRS•TyrSA structure with TyrSA molecule in pink (PDB: 2PID). The absence of the EP is indicated with a dashed arrow. D) HsCytoTyrRS•Tyrosine structure with tyrosine molecule with green carbon atoms (PDB: 4QBT). The lack of the EP is indicated with a dashed arrow. E) Surface representation of $L d$ TyrRS binding pocket with bound TyrSA molecule with purple carbon atoms. Protein carbon atoms are colored grey, nitrogens blue, oxygens red, and sulfur atoms yellow. The adenine binding pocket (ABP) and EP are indicated. The three water molecules occupying the EP are shown as red spheres. Two of those (Wat1 and Wat2) are deeply positioned in the EP. F) Surface representation of $H s$ MitoTyrRS binding pocket in with bound TyrSA molecule with pink carbon atoms (PDB: 2PID) in the same orientation as the $L d$ TyrRS structure in E. Like in the human cytosolic enzyme binding pocket (not shown in surface representation), no EP is present. 


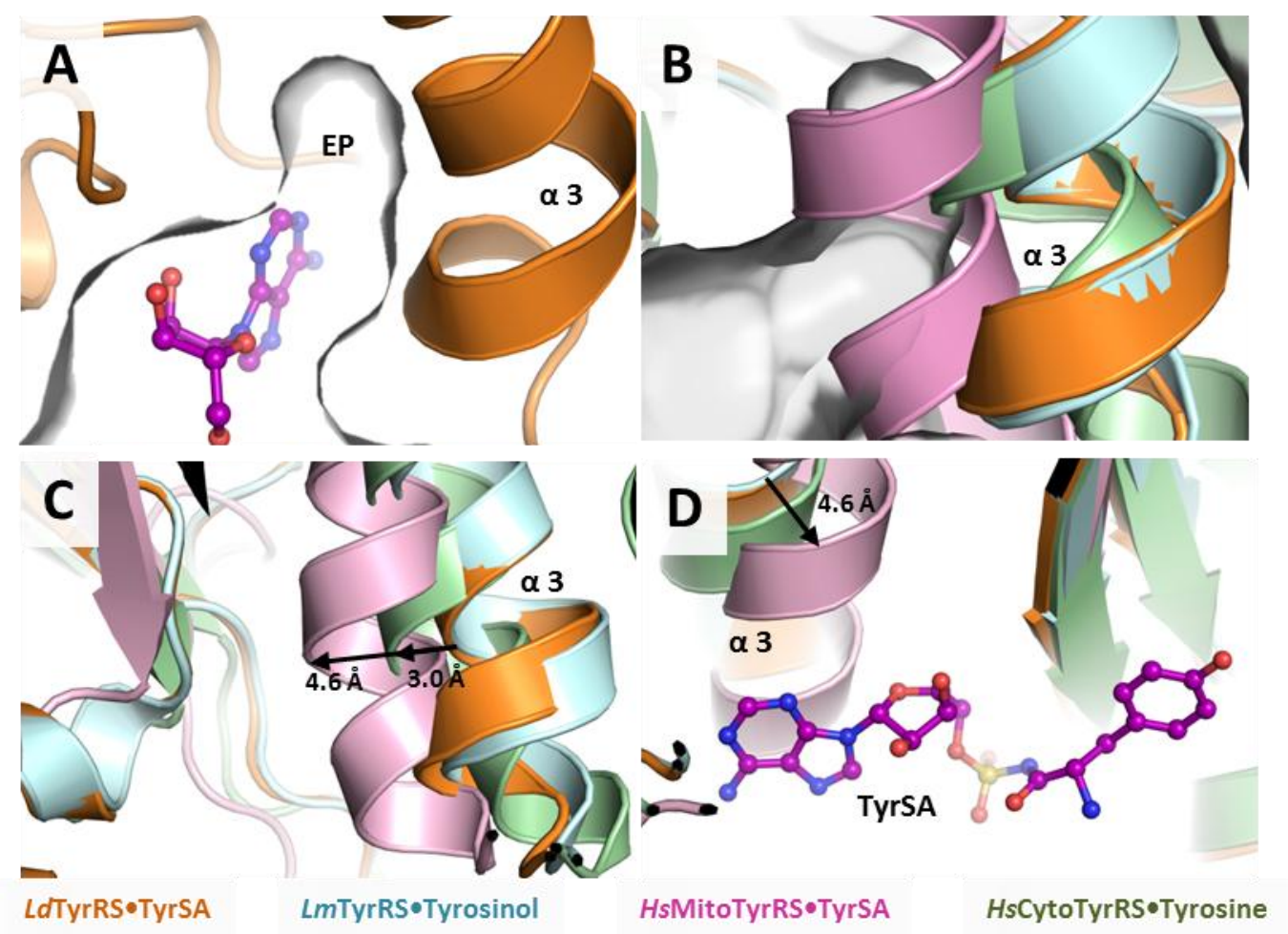

Figure 8. Comparison of $\boldsymbol{\alpha} 3$ in human and Leishmania TyrRS structures. The superposition of structures was done to understand the absence of the EP in the human TyrRSs. A) LdTyrRS TyrSA structure in orange with TyrSA molecule in purple showing the EP surface in grey. B) Superposition of $L d$ TyrRS•TyrSA with $H s$ MitoTyrRS•TyrSA (PDB: 2PID) in pink, HsCytoTyrRS•Tyrosine (PDB: 4QBT) in light green and LmTyrRS•Tyrosinol (PDB: 3POJ) in cyan; LdTyrRS EP surface in grey. C) A $4.6 \AA$ and $3.0 \AA$ shift in $\alpha 3$ prevents the formation of the EP in $H s$ MitoTyrRS and $H s$ CytoTyrRS, respectively. D) Another view of $\alpha 3$ superposition and its shift in $H s$ TyrRS structures. 
It is worthwhile to try to discern why the EP is absent in the human enzymes. It appears that helix $\alpha 3$ side chains forming the EP in $L d$ TyrRS are all different in the human enzymes. For instance, Q49 of LdTyrRS is equivalent to Y52 and H91 in the human cytosolic and mitochondrial enzymes, F52 of LdTyrRS to V54 and L93 in these two human homologs, K53 of LdTyrRS to P55 and A94, and N56 of LdTyrRS to K58 and G97, respectively. Actually, the $L$. donovani TyrRS residues from C35 to K59, comprising helix $\alpha 3$, and the L. donovani TyrRS residues V206 to L213, surrounding $L d$ TyrRS H210, are essentially all different when comparing LdTyrRS to the two human TyrRS enzymes (Figure 9). As a result of all these changes, helix $\alpha 3$ shifts in the human enzymes compared to the L. donovani enzyme, and the EP is absent in the human homologs (Figure 7).

\subsection{A sequence fingerprint for the extra pocket (EP)}

The seven residues forming the EP in LdTyrRS: D37, A48, Q49, F52, K53, N56 and H210 can be used as an "EP fingerprint": D-x $10-\mathrm{AQ}-\mathrm{x}_{2}-\mathrm{FK}-\mathrm{x}_{2}-\mathrm{N}-\mathrm{z}-\mathrm{H}$, where $\mathrm{x}$ stands for any amino acid and $\mathrm{z}$ for a variable large number of residues. This EP fingerprint is present in the TyrRS sequences of all Leishmania species analyzed (Figure 9). Hence, it is likely that the EP is present in all Leishmania TyrRS enzymes, as confirmed by a comparison of the L. major and $L$. donovani TyrRS structures (Figure 7 A,B). The EP fingerprint also occurs in other Trypanosomatids. The latter include the important human pathogens Trypanosoma brucei and $T$. cruzi, as well as T. vivax, causing nagana in cattle in sub-Saharan Africa. Given the absence of the EP in the two human homologs, this difference indicates interesting opportunities for arriving at compounds with higher affinity for Trypanosomatid than for the human tyrosyl-tRNA synthetases. 
Helix 3

\begin{tabular}{|c|c|c|c|}
\hline & & 50 & 210 \\
\hline Ldonovani & : & LIRCYDGEPSGR-MHIA CIER ANPNKCT. & VILSHAML. \\
\hline Lmajor & : & LIRCYDEFEPSGR-MHIA EIIERNNNNKCT. & VILSHAML. \\
\hline Lmexicana & : & LIRCYDEEEPSGR-MHIACEVERANVNKCT. & VILSHHML. \\
\hline Tcruzi & : & NIRCYDEEEPSGR-MHIAGEVERVNONKCT & VILSHHML \\
\hline Tbrucei & : & DIRCYDEEERSG-MHVACEREINVNKCT & VILSHHML. \\
\hline Tvivax & : & SIRCYLEFEPSGR-VHIAEGVERAINNKCT & VILSHHML. \\
\hline Pfalciparum & : & KLICYDEFEPSGR-MHIAEGLESIIVNKLT & VILSHGML \\
\hline Pvivax & : & RLVCYDEEEPSGR-MHIA ELLREIVNKLT & IILSHCML \\
\hline Cparvum & : & HPICYDGEEPGR-MHIA EILEINPNKLT & VILSHKML \\
\hline Tgondii & : & FPLCYDEEEPSGR-MHVACELRVINVNKLT & VILSHCML \\
\hline Glamblia & : & RLTCYDGEESGR-IHIACEIAKANNNRLL & VILMHAML \\
\hline Ehistolytica & : & NEVAYNGEEPSGR-IHIAGAILTVINANKIH & IILSHHML: \\
\hline HsapCyto & : & ELKIYWGTATTGK-PHVAY-FVPMSKIADFL & VHLMNPMV \\
\hline HsapMito & : & PQTIYCGEDPTADSLHVGH-LLALLGLFHLQ & EGITVPLI \\
\hline Scerevisiae & : & HLKLYWGTAPTGR-PHCGY-FVPMTKLADEL & AHLMNPMV \\
\hline Saureus & : & QVTLYCGADPTADSLHIGH-LLEFLTLRREQ & YGLTIPLV \\
\hline Mtuberculosis & : & PMTVYAGEDPTAPSLHAGH-LVPLLTLRREQ & HALTVPLV \\
\hline Mjannaschii & : & EKSAYIGEEPSGK-IHLGH-YLQIKKMIDLQ & VCIHNPVL \\
\hline
\end{tabular}

Figure 9. Amino acid sequence alignment of selected species and the signature fingerprint of the extra pocket (EP). The residues involved in the EP formation in L. donovani (top line) are indicated with red boxes. When the corresponding residues in other species are identical to those of the EP fingerprint in $L d$ TyrRS then these residues are also enclosed in a red box. Residues L and I at positions 4 and 6 of the $L d$ TyrRS EP fingerprint are indicated with orange boxes. The EP was not found in the available structures of human, yeast, bacteria or archaeal (Methanocaldococcus jannaschii [49]) TyrRSs which agrees with the lack of conservation of the EP fingerprint residues. The amino acid sequence alignment of $L d$ TyrRS with enzymes from selected pathogenic bacterial species shows that these bacterial TyrRS do not contain an EP, and comparisons of TyrRS crystal structures from (S. aureus and $M$. tuberculosis [20,48] ) with $L d$ TyrRS confirm that this indeed the case (not shown). The full species names are $:$ Ldonovani $=$ Leishmania donovani $;$ Lmajor $=$ Leishmania major $;$ Lmexicana $=$ Leishmania mexicana $;$ Tcruzi $=$ Trypanosoma cruzi ; Tbrucei = Trypanosoma brucei Pfalciparum = Plasmodium falciparum ; Pvivax = Plasmodium vivax ; Cparvum $=$ Cryptosporidium parvum $;$ Tgondii $=$ Toxoplasma gondii $;$ Glamblia $=$ Giardia lamblia $;$ Ehistolytica $=$ Entamoeba hystolitica $;$ HsapCyto $=$ cytosolic Homo sapiens; HsapMito $=$ mitochondrial Homo sapiens $;$ Scerevisiae $=$ Saccharomyces cerevisiae $;$ Saureus $=$ Staphylococcus aureus $;$ Mtuberculosis $=$ Mycobacterial tuberculosis; Mjannaschii = Methanocaldococcus jannaschii . 


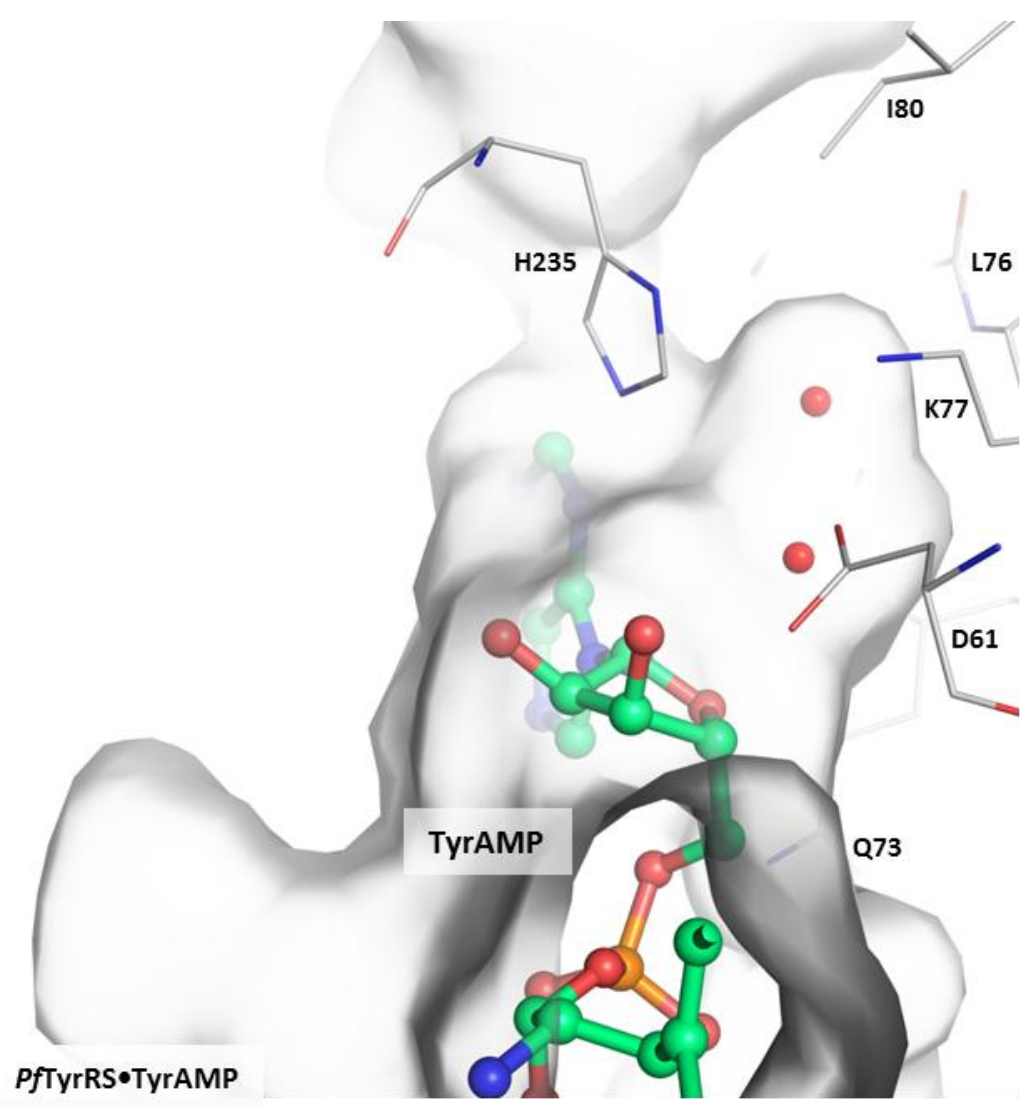

Figure 10. P. falciparum TyrRS residues forming the extra pocket (EP). Surface representation of the EP in the PfTyrRS•TyrAMP crystal structure [29] (PDB: 3VGJ). Protein carbon atoms are colored grey, nitrogens blue, oxygens red and phosphorous orange. All but two (L76 and I80) of the residues involved in the EP formation are shared with trypanosomatids. PfTyrRS harbors one deep water in the EP (equivalent to Wat2 in $L d$ TyrRS) and a water molecule at the EP entrance (equivalent to Wat 3 in $L d$ TyrRS), shown as red spheres. TyrAMP carbon atoms are shown in green. 


\section{Discussion}

The structure of the $75 \mathrm{kDa} L d$ TyrRS in complex with TyrSA has increased our insights in the architecture of this unusual member of the tRNA synthetase family. The two pseudomonomers contain each two domains, in the N-terminal pseudo-monomer a functional catalytic domain and a non-functional anticodon-binding domain, and in the C-terminal pseudo-monomer a non-functional catalytic domain and a functional anticodon binding domain. A similar architecture had been observed for L. major TyrRS [27]. Comparison of the two crystallographically independent chains in our structure showed that the two pseudo-monomers can flex with respect to each other. This results in a considerable difference in position of the functional anti-codon binding domain at the C-terminus of the second pseudo-monomer when the N-terminal pseudo-monomers are superimposed (Figure 2). This flexibility might be essential in positioning tRNA ${ }^{T y r}$ properly with respect to the catalytic domain during the second catalytic step of the reaction catalyzed by Leishmania TyrRS.

The binding mode of TyrSA to LdTyrRS provides additional insight as to how the tyrosyl-adenylate adduct binds to the active site of this group of TyrRS enzymes. A new feature revealed by our analysis of the $L d$ TyrRS structure is the presence of an "extra pocket" (EP), close to the adenine binding pocket and filled by three mutually interacting water molecules. This EP is present as well in the L. major TyrRS structure [27] and, based on amino acid sequence comparisons, also in other Trypanosomatids. Several of the latter, such a T. brucei and T. cruzi, are major global human pathogens causing death and disease in particular in lowincome countries [2, 3]. Since the EP is absent in the two human TyrRS enzymes, this difference between enzymes from parasite and human host indicates opportunities to arrive at inhibitors with high affinity and selectivity.

It is of interest to evaluate if the EP is present in the TyrRS from other medically important parasites. The enzyme from the protozoan Plasmodium falciparum, the major causative agent of malaria, has been the subject of several studies, including the determination of the crystal structure of cytosolic P. falciparum TyrRS (PfTyrRS) [29]. The EP fingerprint is present in PfTyrRS with two changes: the F at the fourth position in the fingerprint is in PfTyrRS an $\mathrm{L}$, which conserves the hydrophobic side chain characteristic at this position, and the $\mathrm{N}$ at the sixth position is an I in PfTyrRS (Figure 9). A comparison of the structure of $L d$ TyrRS with the crystal structure of P. falciparum TyrRS [29], reveals that in the latter enzyme the EP is indeed 
present (Figure 10). In this case, the EP harbors only one deep water molecule, the equivalent of Wat 2 in the $L d$ TyrRS EP. The absence of Wat1 is due to the fact that residue N56, in the back of the EP of $L d$ TyrRS (Figure 7 B), is substituted by I80 in the P. falciparum enzyme, providing a more hydrophobic environment within the pocket and no hydrogen-bonding partner for Wat1. Based on the amino acid sequence alignment (Figure 9), this same feature is present in $P$. vivax, another important malaria parasite. These comparisons suggest that there are opportunities to arrive at high affinity PfTyrRS inhibitors with good selectivity. This is extra interesting since Kahn [18] has recently emphasized the opportunities of TyrRS as a drug target for malaria.

Turning to other protozoa causing considerable human suffering and death across the globe, the comparison of EP fingerprints in Cryptosporidium parvum, Toxoplasma gondii and Giardia lamblia reveals that in these pathogens there is only a single residue difference in the fingerprint: the $\mathrm{F}$ at the fourth position is an L, I or A in these three parasites, respectively. Hence the EP in these protozoa is even closer to that of $L d$ TyrRS than the EP in the two $P$. falciparum species. The $\mathrm{N}$ at the sixth position of the fingerprint is maintained so that it is very likely that in these three species the EP is present with Wat1 deep in the pocket. Consequently, the EP might enable the development of effective and selective TyrRS inhibitors for treating cryptosporidiosis, toxoplasmosis and giardiasis. Although in Entamoeba histolytica the EP fingerprint is less perfectly conserved, with differences in the first ( $D$ to $N$ ), fourth ( $F$ to L) and fifth (K to $\mathrm{T}$ ) positions with respect to $L d \mathrm{TyrRS}$, two of these three side chain changes are very conservative. Hence, an exploitable EP might also exist in the TyrRS of this pathogen.

\section{Conclusions}

The structure of the $L d \mathrm{TyrRS} \bullet \mathrm{NbA} \bullet \mathrm{TyrSA}$ complex elucidated revealed different mutual orientations of its two pseudo-monomers. The comparison of the LdTyrRS structure to both human homologs showed major differences compared in the active site, in particular the presence of an "extra pocket" in the parasite enzyme. The latter feature is also found in the TyrRSs from other unicellular parasites and could be a key element in the development of novel compounds for treating diseases caused by a wide range of important pathogenic protozoa. 


\section{Acknowledgments}

We like to thank Ethan Merritt for stimulating discussions. Research reported in this publication was supported by the National Institute of Allergy and Infectious Diseases of the National Institutes of Health under award number R01AI084004 (to WGJH) and R01AI097177 (to FSB and EF). We thank Instruct, part of the European Strategy Forum on Research Infrastructures (ESFRI), and the Research Foundation Flanders (FWO) for their support to the Nanobody discovery. We are grateful to Nele Buys for the technical assistance during Nanobody discovery. We acknowledge the support of a Fulbright Fellowship to X.B.-A. We thank Robert Steinfeldt for providing support for computing environment at the Biomolecular Structure Center of the University of Washington. Crystallography performed in support of the work benefitted from remote access to resources at the Stanford Synchrotron Radiation Lightsource supported by the U.S. Department of Energy Office of Basic Energy Sciences under Contract No. DE-AC0276SF00515 and by the National Institutes of Health (P41GM103393). The content is solely the responsibility of the authors and does not necessarily represent the official views of the National Institutes of Health.

\section{Author contributions}

Protein purification and crystallization: KMM, CYK

Nanobody generation: EP and JS

Inhibitor synthesis: ZZ and EF.

Enzyme inhibition measurements: RMR, JRG, FSB

Crystallographic data collection and refinement: XB-A, ST

Structure analysis: XB-A, CLMJV, WGJH

$\mathrm{XB}-\mathrm{A}$ and WGJH wrote the manuscript with input from all authors. 


\section{References}

1. DNDi. Leishmaniasis Fact Sheet. 2015; Available from: http://www.dndi.org/wpcontent/uploads/2016/10/Factsheet_2015_Leish.pdf.

2. WHO. Leishmaniasis Fact Sheet, September 2016. 2016; Available from: http://www.who.int/mediacentre/factsheets/fs375/en/.

3. WHO. Control of the Leishmaniasis. . Report of a meeting of the WHO Expert Committee on the Control of Leishmaniases, 22 -- 26 March 2010, Geneva 2010 2010; Available from: http://apps.who.int/iris/bitstream/10665/44412/1/WHO_TRS_949_eng.pdf

4. Fairlamb, A.H., et al., Drug resistance in eukaryotic microorganisms. Nat Microbiol, 2016. 1(7): p. 16092.

5. Sundar, S. and J. Chakravarty, Leishmaniasis: an update of current pharmacotherapy. Expert Opin Pharmacother, 2013. 14(1): p. 53-63.

6. Ibba, M. and D. Soll, Aminoacyl-tRNA synthesis. Annual Review of Biochemistry, 2000. 69: p. 617-650.

7. Sheppard, K., P.M. Akochy, and D. Soll, Assays for transfer RNA-dependent amino acid biosynthesis. Methods, 2008. 44(2): p. 139-45.

8. Charriere, F., et al., Dual targeting of a tRNAAsp requires two different aspartyl-tRNA synthetases in Trypanosoma brucei. J Biol Chem, 2009. 284(24): p. 16210-7.

9. Ochsner, U.A., et al., Aminoacyl-tRNA synthetases: essential and still promising targets for new anti-infective agents. Expert Opin Investig Drugs, 2007. 16(5): p. 573-93.

10. Pham, J.S., et al., Aminoacyl-tRNA synthetases as drug targets in eukaryotic parasites. Int J Parasitol Drugs Drug Resist, 2014. 4(1): p. 1-13.

11. Schimmel, P., J.S. Tao, and J. Hill, Aminoacyl tRNA synthetases as targets for new antiinfectives. Faseb Journal, 1998. 12(15): p. 1599-1609.

12. Fang, P., et al., Structural basis for full-spectrum inhibition of translational functions on a tRNA synthetase. Nat Commun, 2015. 6: p. 6402.

13. Nakama, T., O. Nureki, and S. Yokoyama, Structural basis for the recognition of isoleucyl-adenylate and an antibiotic, mupirocin, by isoleucyl-tRNA synthetase. J Biol Chem, 2001. 276(50): p. 47387-93.

14. Rock, F.L., et al., An antifungal agent inhibits an aminoacyl-tRNA synthetase by trapping tRNA in the editing site. Science, 2007. 316(5832): p. 1759-1761.

15. Koh, C.Y., et al., Structures of Trypanosoma brucei methionyl-tRNA synthetase with urea-based inhibitors provide guidance for drug design against sleeping sickness. PLoS Negl Trop Dis, 2014. 8(4): p. e2775.

16. Khan, S., et al., Structural basis of malaria parasite lysyl-tRNA synthetase inhibition by cladosporin. Journal of Structural and Functional Genomics, 2014. 15: p. 9.

17. Zhou, H., et al., ATP-directed capture of bioactive herbal-based medicine on human tRNA synthetase. Nature, 2013. 494(7435): p. 121-4.

18. Khan, S., Recent advances in the biology and drug targeting of malaria parasite aminoacyl-tRNA synthetases. Malar J, 2016. 15: p. 203. 
19. Greenwood, R.C. and D.R. Gentry, Confirmation of the antibacterial mode of action of $S B-219383$, a novel tyrosyl tRNA synthetase inhibitor from a Micromonospora sp. Journal of Antibiotics, 2002. 55(4): p. 423-426.

20. Qiu, X., et al., Crystal structure of Staphylococcus aureus tyrosyl-tRNA synthetase in complex with a class of potent and specific inhibitors. Protein Science, 2001. 10: p. 9.

21. Stefanska, A.L., et al., SB-219383, a novel tyrosyl tRNA synthetase inhibitor from a Micromonospora sp I. Fermentation, isolation and properties. Journal of Antibiotics, 2000. 53(4): p. 345-350.

22. Brown, P., et al., Synthetic analogues of SB-219383. Novel C-glycosyl peptides as inhibitors of tyrosyl tRNA synthetase. Bioorganic \& Medicinal Chemistry Letters, 2001. 11(5): p. 711-714.

23. Chen, L.W., et al., Metronidazole containing pyrazole derivatives potently inhibit tyrosyltRNA synthetase: design, synthesis, and biological evaluation. Chem Biol Drug Des, 2016. 88(4): p. 592-8.

24. Jarvest, R.L., et al., Interaction of tyrosyl aryl dipeptides with S-aureus tyrosyl tRNA synthetase: Inhibition and crystal structure of a complex. Bioorganic \& Medicinal Chemistry Letters, 1999. 9(19): p. 2859-2862.

25. Wang, X.D., et al., 3-Aryl-4-acyloxyethoxyfuran-2(5H)-ones as inhibitors of tyrosyl-tRNA synthetase: Synthesis, molecular docking and antibacterial evaluation. Bioorganic \& Medicinal Chemistry, 2013. 21(17): p. 4914-4922.

26. Anand, S. and R. Madhubala, Twin Attributes of Tyrosyl-tRNA Synthetase of Leishmania donovani: A HOUSEKEEPING PROTEIN TRANSLATION ENZYME AND A MIMIC OF HOST CHEMOKINE. J Biol Chem, 2016. 291(34): p. 17754-71.

27. Larson, E.T., et al., The double-length tyrosyl-tRNA synthetase from the eukaryote Leishmania major forms an intrinsically asymmetric pseudo-dimer. J Mol Biol, 2011. 409(2): p. 159-76.

28. Pardon, E., et al., A general protocol for the generation of Nanobodies for structural biology. Nat Protoc, 2014. 9(3): p. 674-93.

29. Bhatt, T.K., et al., Malaria parasite tyrosyl-tRNA synthetase secretion triggers proinflammatory responses. Nat Commun, 2011. 2: p. 530.

30. Shibata, S., et al., Selective inhibitors of methionyl-tRNA synthetase have potent activity against Trypanosoma brucei Infection in Mice. Antimicrob Agents Chemother, 2011. 55(5): p. 1982-9.

31. Shibata, S., et al., Urea-based inhibitors of Trypanosoma brucei methionyl-tRNA synthetase: selectivity and in vivo characterization. J Med Chem, 2012. 55(14): p. 634251.

32. Pedro-Rosa, L., et al., Identification of potent inhibitors of the Trypanosoma brucei methionyl-tRNA synthetase via high-throughput orthogonal screening. J Biomol Screen, 2015. 20(1): p. 122-30.

33. Otwinowski, Z. and W. Minor, Processing of X-ray diffraction data collected in oscillation mode, in Macromolecular Crystallography, Part A, C. Carter and R. Sweet, Editors. 1997 Academic Press. p. 307-326.

34. McCoy, A.J., et al., Phaser crystallographic software. J Appl Crystallogr, 2007. 40(Pt 4): p. 658-674.

35. Kelley, L.A., et al., The Phyre2 web portal for protein modeling, prediction and analysis. Nat Protoc, 2015. 10(6): p. 845-58. 
36. Emsley, P., et al., Features and development of Coot. Acta Crystallogr D Biol Crystallogr, 2010. 66(Pt 4): p. 486-501.

37. Murshudov, G.N., A.A. Vagin, and E.J. Dodson, Refinement of macromolecular structures by the maximum-likelihood method. Acta Crystallogr D Biol Crystallogr 1997. 53: p. 16.

38. Smart, O.S., et al. Grade Web Server, version 1.001. . 2011; Available from: http://grade.globalphasing.org.

39. Chen, V.B., et al., MolProbity: all-atom structure validation for macromolecular crystallography. Acta Crystallogr D Biol Crystallogr, 2010. 66(Pt 1): p. 12-21.

40. Schrödinger, L.L.C. The PyMOL Molecular Graphics System, Version 1.7. . Available from: https://www.pymol.org.

41. Kobayashi, T., et al., Structural snapshots of the KMSKS loop rearrangement for amino acid activation by bacterial tyrosyl-tRNA synthetase. J Mol Biol, 2005. 346(1): p. 105-17.

42. Tsunoda, M., et al., Structural basis for recognition of cognate tRNA by tyrosyl-tRNA synthetase from three kingdoms. Nucleic Acids Res, 2007. 35(13): p. 4289-300.

43. Krissinel, E. and K. Henrick, Inference of macromolecular assemblies from crystalline state. J Mol Biol, 2007. 372(3): p. 774-97.

44. Fricker, P., M. Gastreich, and M. Rarey, Automated Generation of Structural Molecular Formulas under Constraints. Journal of Chemical Information and Computer Sciences, 2004. 44(3): p. 14.

45. Stierand, K., P.C. Maass, and M. Rarey, Molecular complexes at a glance: automated generation of two-dimensional complex diagrams. Bioinformatics, 2006. 22(14): p. 17106.

46. Sajish, M. and P. Schimmel, A human tRNA synthetase is a potent PARP1-activating effector target for resveratrol. Nature, 2015. 519(7543): p. 370-+.

47. Bonnefond, L., et al., Crystal structure of human mitochondrial tyrosyl-tRNA synthetase reveals common and idiosyncratic features. Structure, 2007. 15(11): p. 1505-1516.

48. Hartmann, M.D., et al., The Structure of Tyrosyl-tRNA Synthetase from Mycobacterium Tuberculosis, PDB ID: 2JAN. 2006.

49. Kobayashi, T., et al., Structural basis for orthogonal tRNA specificities of tyrosyl-tRNA synthetases for genetic code expansion. NATURE STRUCTURAL BIOLOGY 2003. 10 (6). 


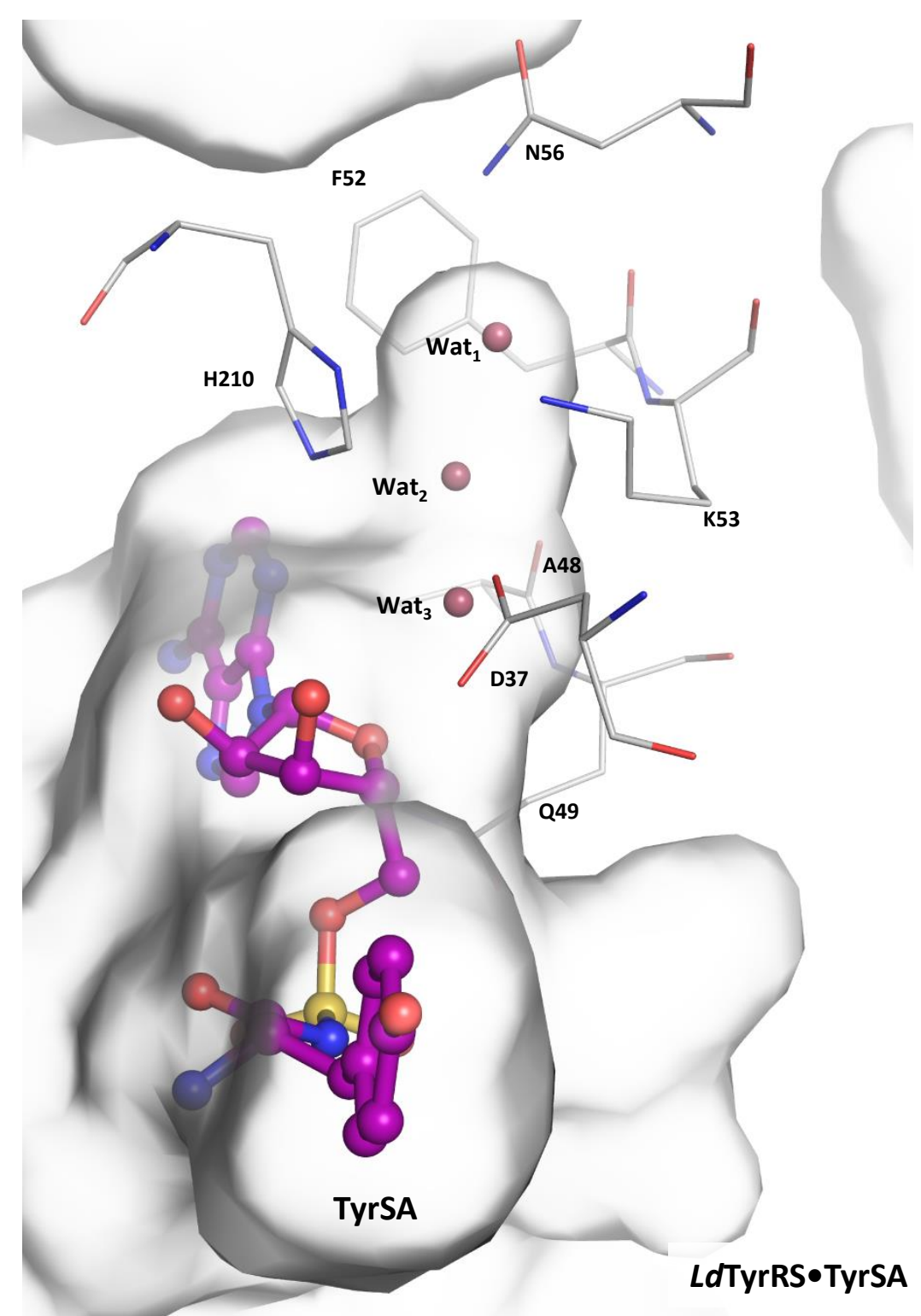

